\title{
Three-Dimensional Imaging and Scattering Mechanism Estimation Over Urban Scenes Using Dual-Baseline Polarimetric InSAR Observations at L-Band
}

\author{
Stefan Sauer, Laurent Ferro-Famil, Member, IEEE, \\ Andreas Reigber, Senior Member, IEEE, and Eric Pottier, Fellow, IEEE
}

\begin{abstract}
This paper introduces new polarimetric algorithms for generating 3-D images and estimating scattering mechanisms from polarimetric multibaseline (MB) interferometric synthetic aperture radar (SAR) measurements. First, an MB interferometric SAR signal model is generalized to the fully polarimetric configuration, establishing the notion of polarimetric reflectivity. Subsequently, polarimetric beamforming, Capon, and MUSIC methods that determine optimal polarization combinations for height estimation are developed. These new techniques allow for extracting the height of reflectors, the associated scattering mechanisms, and the polarimetric (pseudo)reflectivities. By means of polarimetric dual-baseline interferometric SAR observations of an urban environment, the performance of the conceived algorithms is examined in detail. Producing 3-D images of a building layover, the quality of the approaches is compared in terms of refined resolution and lowered side lobes. Furthermore, the scattering processes occurring in urban scenes are investigated thoroughly by analyzing the optimal reflection types. The algorithms are validated using dual-baseline polarimetric SAR interferometric data at L-band acquired by German Aerospace Center's experimental SAR system over Dresden city.
\end{abstract}

Index Terms-Multibaseline (MB) polarimetric synthetic aperture radar (SAR) interferometry (POL-InSAR), multichannel SAR, polarimetric array signal processing techniques, 3-D polarimetric SAR tomography, 3-D SAR urban remote sensing.

\section{INTRODUCTION}

$\mathbf{T}$ HE ANALYSIS of synthetic aperture radar (SAR) data of urban areas (see [1]-[11] and the references therein) is a very difficult task due to three main reasons.

1) Radar images are geometrically distorted by layover and shadow effects. The layover yields a superposition of different reflection components.

Manuscript received February 13, 2009; revised September 22, 2009, May 5, 2010, and September 30, 2010; accepted March 20, 2011. Date of publication June 16, 2011; date of current version October 28, 2011.

S. Sauer and A. Reigber are with the Microwaves and Radar Institute (HR), German Aerospace Center (DLR), 82234 Oberpfaffenhofen, Germany (e-mail: stefan.sauer@dlr.de; andreas.reigber@dlr.de).

L. Ferro-Famil and E. Pottier are with the SAPHIR, Institute of Electronics and Telecommunications of Rennes, University of Rennes 1, 35042 Rennes, France (e-mail: laurent.ferro-famil@univ-rennes1.fr; eric.pottier@ univ-rennes1.fr).

Color versions of one or more of the figures in this paper are available online at http://ieeexplore.ieee.org.

Digital Object Identifier 10.1109/TGRS.2011.2147321
2) Scattering patterns are very complex with various contributions within one resolution cell. In urban scenes, particular phenomena like single-bounce reflection from surfaces such as building roofs and sport fields, doublebounce scattering generated by building wall-ground, and tree-trunk-ground interaction arise. Also higher order reflection processes may occur.

3) SAR images are affected by the speckle effect that is normally modeled as a multiplicative noise.

Advanced multichannel SAR concepts have been proposed to resolve these problems: Whereas SAR interferometry (InSAR) allows the extraction of the topographic height of scatterers [12], [13], the physics of reflection processes [14][16] can be examined through SAR polarimetry (POLSAR). In polarimetric InSAR (POL-InSAR), both techniques are fused to determine the vertical location of scattering mechanisms [17]-[19]. In [20] and [21], polarization coherence tomography has been introduced, which is a 3-D radar imaging technique for vegetation by means of single- and dual-baseline polarimetric interferometric SAR measurements. SAR tomography constitutes an expansion of traditional 2-D SAR imaging with the objective to reconstruct a 3-D scatterer distribution. An airborne multibaseline (MB) interferometric SAR experiment comprising 14 parallel tracks has been conducted [22], [23] to generate 3-D SAR images and to study scattering patterns via polarimetry.

Recently, array signal processing methods for analyzing single-polarization MB InSAR measurements have been investigated exhaustively by simulations [24]-[26]. They estimate the signal parameters like their spatial frequencies and reflectivities. Determining the spatial frequency or phase results in the height of scatterers. Hence, spectral analysis algorithms resolve the layover problem by retrieving the properties of multiple reflecting sources inside the same azimuth-range resolution element [24]. The reflectivity estimates produce 3-D images [25]. Standard beamforming [27] and Capon [24], [28] belong to nonparametric approaches to extract both phase and amplitude. This means that no assumptions are made about the statistical characteristics of the returned signal. MUSIC [24], [29] is a parametric method for calculating the spatial frequency and the pseudoreflectivity. As this algorithm disregards the speckle effect in the InSAR case, it operates under model mismatch. 
Nevertheless, MUSIC yields generally better estimation quality than nonparametric techniques in terms of spatial resolution and side lobe reduction. For this reason, it has been utilized to generate 3-D images [30], although it is not appropriate to determine reflectivities, but retrieves pseudoreflectivities instead. These techniques have been employed for producing 3-D images by means of single-polarization dual-baseline InSAR measurements [30], [31] and polarimetric tomographic SAR observations [32]-[35]. The ESPRIT method [36] has been suggested to study forested terrain using polarimetric singlebaseline InSAR data and applied to analyze urban areas [37]. Experiments on differential SAR tomography employing spaceborne acquisitions have been reported lately (see [38] and [39] and the references therein).

In [40]-[42], two polarimetric spectral analysis techniques, namely, the MUSIC algorithm and a maximum likelihood estimator (MLE), have been presented to estimate the phases from polarimetric MB InSAR data. In urban environments, the ground and building height has been determined by means of MUSIC and MLE pseudospectra.

This paper establishes a new way of generating 3-D images and estimating scattering mechanisms from polarimetric $\mathrm{MB}$ interferometric SAR measurements by generalizing beamforming, Capon, and MUSIC to this case. These algorithms have been described for the specific setup of polarization diversity in the receive channel [29], [43]. However, in the framework of SAR, the sensors are active. Therefore, wave polarimetry including two polarization channels is augmented to scattering polarimetry incorporating four polarization states. First, the single-polarization MB InSAR signal model is adapted to the polarimetric scenario. The polarimetric MB interferometric steering vector is a linear combination of four vectors, each of them corresponding to one particular polarization. The coefficients of this combination compose a polarimetric reflection vector that permits the retrieval of the scatterer physical properties. Moreover, the concept of the polarimetric reflectivity is introduced. Subsequently, polarimetric array signal processing algorithms are conceived in a rigorous mathematical manner, and their characteristics are described. The adaptation to the polarimetric configuration not only increases the number of observables but also particularly computes the optimal polarization combination for spatial frequency estimation. Furthermore, they enable the recovery of physical features of the scatterers by carrying out a polarimetric investigation of the determined reflection types. In addition, the polarimetric (pseudo)reflectivities can be calculated.

The developed spectral analysis techniques are applied to polarimetric and repeat-pass interferometric SAR data with two baselines at L-band to reveal their performances. The data set was acquired by DLR's experimental SAR (E-SAR) system [44] over an urban scene. A building layover is scrutinized by generating 3-D images that include polarimetric information about the reflection processes. The qualities of beamforming, Capon, and MUSIC are compared with one another in terms of spectral width and leakage lowering. In particular, the performance disparities of the single-polarization and fully polarimetric approaches are pointed out. Moreover, the scattering mechanisms are investigated thoroughly for a complex urban area.
The main novel contributions of this paper can be recapitulated by the following points: A new data model including the polarimetric MB interferometric steering vector and the polarimetric reflectivity is introduced. Three array signal processing techniques are extended to the polarimetric case to estimate the polarimetric (pseudo)reflectivity and the reflection mechanisms. After examining the estimation accuracy of the single-polarization and polarimetric techniques, 3-D images are produced, and scattering mechanisms are analyzed in complex urban scenes.

The remainder of this paper is organized as follows: In Section II, a standard single-polarization signal model and the conventional beamforming, Capon, and MUSIC techniques are described. Section III presents the expansion to the fully polarimetric MB InSAR constellation: First, the signal model is adapted to compass four polarization channels. Subsequently, the polarimetric methods are devised in a rigorous mathematical way, and their properties are defined. Finally, experimental results are displayed in Section IV. This paper finishes with discussion and conclusion in Section V.

\section{Classical Array Signal Processing Techniques}

\section{A. MB InSAR Signal Model}

An established signal model of single-polarization $\mathrm{MB}$ InSAR data with $p$ antennas [24]-[26] contains the SAR speckle phenomenon as multiplicative noise. For extended sources, the measurement vector $\mathbf{y}(l)$ is expressed as

$$
\begin{aligned}
\mathbf{y}(l) & =\mathbf{c}(l)+\mathbf{n}(l) \\
& =\sum_{j=1}^{N_{s}} \sqrt{\tau_{j}} \mathbf{x}_{j}(l) \odot \mathbf{a}\left(\theta_{j}\right)+\mathbf{n}(l)
\end{aligned}
$$

with the number of independent realizations $L, l=1, \ldots, L$, and the Schur-Hadamard product $\odot$ (elementwise multiplication). The term $\mathbf{c}(l)$ incorporates the SAR speckle effect [24][26] as multiplicative noise $\mathbf{x}_{j}(l)$ of source $j$ and represents the response of natural targets. The additive noise is denoted by $\mathbf{n}(l)$, and the steering vector is denoted by $\mathbf{a}\left(\theta_{j}\right)$. The number of backscattering sources $N_{s}$ is assumed to be known. The reflectivity $\tau_{j}$ is unknown and considered to be a deterministic parameter.

For single-polarization MB interferometric SAR observations, the vector $\mathbf{a}\left(\theta_{j}\right)$ possesses the following shape: The steering vector $\mathbf{a}(\varphi) \in \mathbb{C}^{p}$ of a uniform linear array (ULA) depends on the interferometric phase at the overall baseline $\varphi$, i.e., the phase difference between the two farthest phase centers

$$
\mathbf{a}(\varphi)=[1, \exp \{j \varphi /(p-1)\}, \ldots, \exp \{j \varphi\}]^{\mathrm{T}} .
$$

The phase $\varphi$ corresponds to the spatial frequency $\omega, \omega \in$ $[-\pi, \pi)$, via [24], [45], [46]

$$
\varphi=(p-1) \omega .
$$

For an irregular track distribution, i.e., a general acquisition geometry, the steering vector $\mathbf{a}(z)$ is a function of the scatterer 


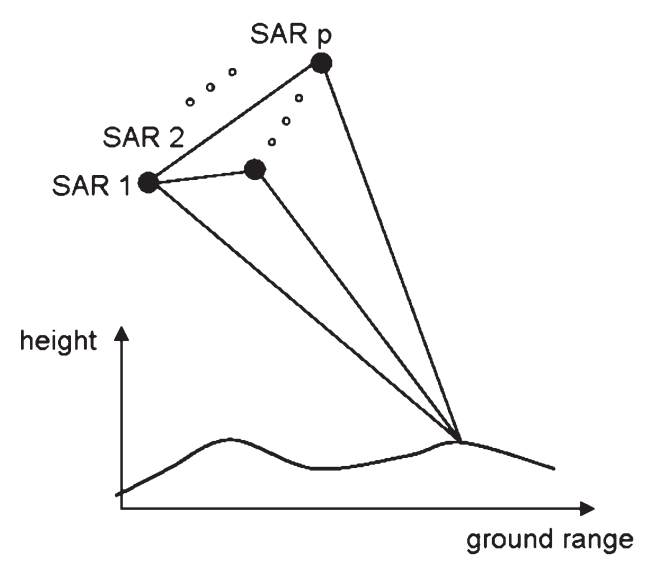

Fig. 1. General MB InSAR imaging geometry.

height $z$ and can be written as

$$
\mathbf{a}(z)=\left[1, \exp \left\{j \kappa_{z_{2}} z\right\}, \ldots, \exp \left\{j \kappa_{z_{p}} z\right\}\right]^{\mathrm{T}} .
$$

The vertical wavenumber is given by $\kappa_{z_{i}}=$ $(4 \pi / \lambda)\left(B_{\perp}^{i} / r^{i} \sin \theta^{i}\right)$, where $\lambda, B_{\perp}, r$, and $\theta$ are the radar wavelength, the orthogonal baseline, the slant range, and the incidence angle, respectively. An MB InSAR system of a nonuniform and nonlinear array is shown in Fig. 1.

The multiplicative noise $\mathbf{x}_{j}(l) \in \mathbb{C}^{p}$ associated with the $j$ th source is modeled as a stationary circular Gaussian-distributed random variable with zero mean, unit variance, and covariance matrix $\mathbf{C}_{j}=E\left\{\mathbf{x}_{j}(l) \mathbf{x}_{j}^{H}(l)\right\}$. The random processes $\mathbf{x}_{j}\left(l_{1}\right)$ and $\mathbf{x}_{j}\left(l_{2}\right)$ are supposed to be independent and identically distributed (locally stationary in a homogeneous area) for different looks $l_{1} \neq l_{2}$. The sources can be assumed to be independent when multipath scattering is ignored. The additive noise $\mathbf{n}(l)$ is supposed to be independent of the signals and to be a stationary and ergodic Gaussian random vector with zero mean and covariance matrix $\sigma_{n}^{2} \mathbf{I} \in \operatorname{Mat}_{p}(\mathbb{C})$, where $\sigma_{n}^{2}$ is the unknown noise power. Hence, the stochastic MB InSAR returned signal $\mathbf{y}(l)$ is a Gaussian random vector having zero mean and covariance matrix $\mathbf{R}$ that can be expressed as

$$
\mathbf{R}=E\left\{\mathbf{y}(l) \mathbf{y}^{H}(l)\right\}=\sum_{j=1}^{N_{s}} \tau_{j} \mathbf{C}_{j} \odot \mathbf{a}\left(\theta_{j}\right) \mathbf{a}^{H}\left(\theta_{j}\right)+\sigma_{n}^{2} \mathbf{I} .
$$

The data covariance matrix must be estimated, as it is not known a priori. Assuming a Gaussian distribution, the maximum likelihood estimate of the sample covariance matrix $\hat{\mathbf{R}} \in$ $\operatorname{Mat}_{p}(\mathbb{C})$ is given by

$$
\hat{\mathbf{R}}=\frac{1}{L} \sum_{l=1}^{L} \mathbf{y}(l) \mathbf{y}^{H}(l) .
$$

The objective is to determine the heights $z_{j}$ (or interferometric phases $\varphi_{j}$ ) and the reflectivities $\tau_{j}$.

\section{B. Conventional Beamforming}

The spectrum of the beamforming algorithm is given by [27], [46]

$$
\hat{P}_{\mathrm{BF}}(\omega)=\frac{1}{p^{2} L} \sum_{l=1}^{L}\left|\mathbf{a}^{H}(\omega) \mathbf{y}(l)\right|^{2}=\frac{\mathbf{a}^{H}(\omega) \hat{\mathbf{R}} \mathbf{a}(\omega)}{p^{2}} .
$$

The frequency estimates $\hat{\boldsymbol{\omega}}=\left[\hat{\omega}_{1}, \ldots, \hat{\omega}_{N_{s}}\right]^{\mathrm{T}}$ are associated with the frequencies of the $N_{s}$ highest peaks of the spectrum $\hat{P}_{B F}$. The reflectivity estimate at frequency $\hat{\omega}_{i}$ is determined by $\hat{\tau}_{i}=\hat{P}_{\mathrm{BF}}\left(\hat{\omega}_{i}\right)$. It follows from the first equation in (7) that beamforming does not require spatial averaging $(L=1)$. Nevertheless, it is generally performed to reduce speckle [24], [25].

\section{Standard Capon}

The Capon spectrum is obtained as [28]

$$
\hat{P}_{C}(\omega)=\frac{1}{\mathbf{a}^{H}(\omega) \hat{\mathbf{R}}^{-1} \mathbf{a}(\omega)}
$$

The inverse matrix $\hat{\mathbf{R}}^{-1}$ exists if the noise term has a positive definite covariance matrix and $L \geq p$ [46]. The positions of the $N_{s}$ maxima of the spectrum provide the frequency estimates $\hat{\boldsymbol{\omega}}=\left[\hat{\omega}_{1}, \ldots, \hat{\omega}_{N_{s}}\right]^{\mathrm{T}}$. The reflectivity at frequency $\hat{\omega}_{i}$ is estimated by $\hat{\tau}_{i}=\hat{P}_{C}\left(\hat{\omega}_{i}\right)$.

\section{Classical MUSIC}

Let $N_{s}$ denote the assumed number of sources [24], [26] and $\hat{\mathbf{G}} \in \mathbf{M a t}_{p, p-N_{s}}(\mathbb{C})$ be the matrix of the eigenvectors of the data covariance matrix that span the noise subspace. The pseudospectrum of the single-polarization MUSIC method can be expressed by [29], [47]

$$
\hat{P}_{M U}(\omega)=\frac{1}{\mathbf{a}^{H}(\omega) \hat{\mathbf{G}} \hat{\mathbf{G}}^{H} \mathbf{a}(\omega)} .
$$

The spatial frequencies are extracted as the $N_{s}$ maxima of the pseudospectrum $\hat{P}_{M U}$. The pseudoreflectivity at frequency $\hat{\omega}_{i}$ is retrieved according to $\hat{\tau}_{i}=\hat{P}_{M U}\left(\hat{\omega}_{i}\right)$. The subspace spanned by the noise eigenvectors has to be at least of dimension one $\left(p \geq N_{s}+1\right)$.

\section{Polarimetric Array Signal PROCESSING TECHNIQUES}

Array signal processing methods are extended to the polarimetric MB interferometric SAR constellation in this section in compliance with the following principle: In passive radar systems, the sensors merely receive the signals in polarimetric mode, yielding two polarization states [29], [43]. In the field of SAR, antennas are active and both transmit the electromagnetic waves and receive the echoes in polarization diversity, resulting in a maximum of four polarization channels. This designates the crossover from wave polarimetry comprising two polarization states to scattering polarimetry containing four polarizations. First, the signal model is adapted to the polarimetric scenario, leading to the notion of the MB polarimetric interferometric (MBPI) steering vector and introducing the polarimetric reflectivity. Subsequently, the spectral estimation techniques are extended to process polarimetric MB InSAR measurements. The generalization to the polarimetric configuration not only increments the number of observables but also particularly calculates the polarization combination for phase estimation 
TABLE I

POLARIZATION NUMBER $N_{\text {pol }}$

\begin{tabular}{lc}
\hline \hline Polarization state & $N_{\text {pol }}$ \\
\hline single polarization & 1 \\
dual-polarized & 2 \\
fully polarimetric & 3 \\
quad-polarized & 4 \\
\hline
\end{tabular}

that is optimal in terms of the particular algorithm. These optimal reflection types permit the investigation of the scatterer physical features by studying their polarimetric patterns. Hence, the polarimetric methods allow for extracting the spatial frequencies, the related optimal scattering vectors, and the polarimetric (pseudo)reflectivities. The nonparametric beamforming and Capon techniques and the model-based MUSIC algorithm are generalized to the polarimetric $\mathrm{MB}$ interferometric SAR case.

\section{A. Polarimetric MB InSAR Signal Model}

The polarimetric MB InSAR observations $\mathbf{y}(l)$ acquired by $p$ sensors can be modeled as

$$
\mathbf{y}(l)=\sum_{j=1}^{N_{s}} \sqrt{\tau_{j}} \mathbf{x}_{j}(l) \odot \mathbf{b}\left(\boldsymbol{\theta}_{j}\right)+\mathbf{n}(l)
$$

where $L$ is the number of looks and $l=1, \ldots, L$. The number of backscattering sources $N_{s}$ is supposed to be known. The polarimetric reflectivity of source $j$ is symbolized by $\tau_{j}$, the multiplicative noise by $\mathbf{x}_{j}(l)$, the MBPI steering vector by $\mathbf{b}\left(\boldsymbol{\theta}_{j}\right)$, and the additive noise by $\mathbf{n}(l)$. The polarimetric measurement vector $\mathbf{y}(l)$ is of dimension $\tilde{p}$, where $\tilde{p}$ is the product between the polarization number $N_{\text {pol }}$ and the number of antennas $p, \tilde{p}=p N_{\mathrm{pol}}$. The polarization number $N_{\mathrm{pol}}$ adopts the value $N_{\text {pol }}=3$ for fully polarimetric data with equal cross-polarizations and $N_{\text {pol }}=4$ for quad-polarized setups. The coefficient $N_{\text {pol }}=2$ for dual-polarized measurements, and $N_{\text {pol }}=1$ for standard single-polarization configurations. Table I recapitulates the values of $N_{\text {pol }}$.

In comparison with the single-polarization MB InSAR signal model (1), the major alteration is the shape of the MBPI steering vector $\mathbf{b}\left(\boldsymbol{\theta}_{j}\right), \mathbf{b}\left(\boldsymbol{\theta}_{j}\right) \in \mathbb{C}^{\tilde{p}}$. It is a function of the vector of unknown parameters $\boldsymbol{\theta}_{j}$, that are assumed to be deterministic. The properties of the MBPI steering vector are introduced in the following in the framework of the interferometric phase $\varphi$ for a ULA. For the scenario of an irregular track distribution, the phase has to be replaced by the height $z$. The characteristics of the polarimetric steering vector are described in the context of quad-polarized data, and the modifications with regard to other polarimetric constellations are apparent. The MBPI steering vector is linearly combined by four vectors $\mathbf{a}_{\gamma_{i}}(\varphi) \in \mathbb{C}^{\tilde{p}}$, each of them related to one specific polarization

$$
\begin{aligned}
\mathbf{b}(\varphi, \mathbf{k}) & =k_{1} \mathbf{a}_{\gamma_{1}}(\varphi)+k_{2} \mathbf{a}_{\gamma_{2}}(\varphi)+k_{3} \mathbf{a}_{\gamma_{3}}(\varphi)+k_{4} \mathbf{a}_{\gamma_{4}}(\varphi) \\
& =\left[\begin{array}{l}
k_{1} \mathbf{a}(\varphi) \\
k_{2} \mathbf{a}(\varphi) \\
k_{3} \mathbf{a}(\varphi) \\
k_{4} \mathbf{a}(\varphi)
\end{array}\right]
\end{aligned}
$$

The vector $\mathbf{a}(\varphi)$ represents the conventional single-polarization MB InSAR steering vector. The weighting coefficients $k_{i} \in \mathbb{C}$ create a vector

$$
\mathbf{k}=\left[k_{1}, k_{2}, k_{3}, k_{4}\right]^{\mathrm{T}}
$$

that can be interpreted as a unitary reflection mechanism $\mathbf{k}^{H} \mathbf{k}=1$. The matrix notation of the MBPI steering vector is

$$
\mathbf{b}(\varphi, \mathbf{k})=\mathbf{B}(\varphi) \mathbf{k}
$$

with the steering vector matrix $\mathbf{B}(\varphi) \in \operatorname{Mat}_{\tilde{p}, N_{\mathrm{pol}}}(\mathbb{C})$

$$
\mathbf{B}(\varphi)=\left[\begin{array}{cccc}
\mathbf{a}(\varphi) & \mathbf{0} & \mathbf{0} & \mathbf{0} \\
\mathbf{0} & \mathbf{a}(\varphi) & \mathbf{0} & \mathbf{0} \\
\mathbf{0} & \mathbf{0} & \mathbf{a}(\varphi) & \mathbf{0} \\
\mathbf{0} & \mathbf{0} & \mathbf{0} & \mathbf{a}(\varphi)
\end{array}\right]
$$

The unknown parameter $\tau_{j}$ is called the polarimetric reflectivity of the $j$ th source. While the absolute values of the scattering mechanism coefficients $\left|k_{i}\right|$ in (12) indicate the relative intensities between the polarizations, the reflectivity $\tau$ specifies the power corresponding to a radar target. This power is denoted as SPAN and defined by

$$
S P A N=\left|S_{h h}\right|^{2}+\left|S_{h v}\right|^{2}+\left|S_{v h}\right|^{2}+\left|S_{v v}\right|^{2}
$$

where $S_{p q}$ are the elements of the scattering or Sinclair matrix.

The multiplicative noise $\mathbf{x}_{j}(l) \in \mathbb{C}^{\tilde{p}}$ associated with the $j$ th source is assumed to be a stationary circular Gaussian random process with zero mean, unit variance, and covariance matrix $\mathbf{C}_{j}$. For different looks $l_{1} \neq l_{2}$, the random vectors $\mathbf{x}_{j}\left(l_{1}\right)$ and $\mathbf{x}_{j}\left(l_{2}\right)$ are considered to be independent and identically distributed. The additive white Gaussian noise $\mathbf{n}(l) \in \mathbb{C}^{\tilde{p}}$ is supposed to have zero mean and power $\sigma_{n}^{2}$. Therefore, the polarimetric MB InSAR observations $\mathbf{y}(l)$ are a circular Gaussian random process with zero mean and covariance matrix $\mathbf{R}$. It can be written as

$$
\mathbf{R}=E\left\{\mathbf{y}(l) \mathbf{y}^{H}(l)\right\}=\sum_{j=1}^{N_{s}} \tau_{j} \mathbf{C}_{j} \odot \mathbf{b}\left(\boldsymbol{\theta}_{j}\right) \mathbf{b}^{H}\left(\boldsymbol{\theta}_{j}\right)+\sigma_{n}^{2} \mathbf{I} .
$$

The sample covariance matrix $\hat{\mathbf{R}} \in \operatorname{Mat}_{\tilde{p}}(\mathbb{C})$ is computed by

$$
\hat{\mathbf{R}}=\frac{1}{L} \sum_{l=1}^{L} \mathbf{y}(l) \mathbf{y}^{H}(l) .
$$

The generalization of the model to hybrid systems where the antennas capture different polarizations is evident: Let $N_{s p}$, $N_{d p}, N_{f p}$, and $N_{q p}$ denote the numbers of antennas in singlepolarization, dual-polarized, and fully polarimetric modes with equal and diverse cross-polarizations, respectively. It follows that $\tilde{p}=N_{s p}+2 N_{d p}+3 N_{f p}+4 N_{q p}$. The structure of the MBPI steering vector is clear. For example, a dual-baseline InSAR constellation comprising one antenna that is fully polarimetric having $h h, v v$, and $h v$ polarizations, one dual-polarized antenna with $h h$ and $h v$ polarization channels, and one singlepolarization antenna yields $\tilde{p}=6$.

The task is to retrieve the interferometric phases $\varphi_{j}$ (or the heights $z_{j}$ ), the reflection types $\mathbf{k}_{j}$, and the polarimetric 
reflectivities $\tau_{j}$. For this purpose, polarimetric estimation techniques are elaborated next.

\section{B. Polarimetric Beamforming}

In [43], the beamforming algorithm has been adapted to the special setup of polarization diversity in the receive antennas. The polarimetric beamforming method for analyzing polarimetric MB InSAR data is conceived paralleling the finiteimpulse-response filter design procedure. The MB POL-InSAR steering vector $\mathbf{b}(\omega, \mathbf{k}) \in \mathbb{C}^{\tilde{p}}$ is employed instead of the singlepolarization MB InSAR steering vector $\mathbf{a}(\omega) \in \mathbb{C}^{p}$. The polarimetric beamforming optimization problem is written as

$$
\min _{\mathbf{h}} \mathbf{h}^{H} \mathbf{h} \quad \text { subject to } \quad \mathbf{h}^{H} \mathbf{b}(\omega, \mathbf{k})=1 .
$$

Using quadratic minimization (see [46]), the polarimetric beamforming filter can be stated as

$$
\mathbf{h}_{\mathrm{BF}}^{P}=\frac{\mathbf{b}(\omega, \mathbf{k})}{\mathbf{b}^{H}(\omega, \mathbf{k}) \mathbf{b}(\omega, \mathbf{k})}=\frac{\mathbf{b}(\omega, \mathbf{k})}{p} .
$$

The property of the MBPI steering vector

$$
\mathbf{b}^{H}(\omega, \mathbf{k}) \mathbf{b}(\omega, \mathbf{k})=\mathbf{a}^{H}(\omega) \mathbf{a}(\omega) \sum_{i=1}^{N_{\mathrm{pol}}} k_{i}^{*} k_{i}=p\|\mathbf{k}\|_{2}^{2}=p
$$

that was utilized in the last equation of (19) is obtained from (11). It follows that the power of the filtered signal is

$$
E\left\{\left|y_{F}(l)\right|^{2}\right\}=E\left\{\left|\left(\mathbf{h}_{\mathrm{BF}}^{P}\right)^{H} \mathbf{y}(l)\right|^{2}\right\}=\frac{\mathbf{b}^{H}(\omega, \mathbf{k}) \mathbf{R} \mathbf{b}(\omega, \mathbf{k})}{p^{2}} .
$$

Maximizing it as regards the polarizations $\mathbf{k}$ yields

$$
\max _{\|\mathbf{k}\|_{2}=1} \frac{\mathbf{b}^{H}(\omega, \mathbf{k}) \mathbf{R} \mathbf{b}(\omega, \mathbf{k})}{p^{2}} .
$$

The solution of this maximization problem through (13) is the maximum eigenvalue of the Hermitian matrix $\mathbf{B}^{H}(\omega) \mathbf{R B}(\omega) \in \operatorname{Mat}_{N_{\mathrm{pol}}}(\mathbb{C})$

$$
\mathbf{B}^{H}(\omega) \mathbf{R B}(\omega) \mathbf{k}_{\max }=\lambda_{\max } \mathbf{k}_{\max } .
$$

With the sample covariance matrix $\hat{\mathbf{R}}$, the spectrum of the polarimetric beamforming is given by

$$
\hat{P}_{\mathrm{BF}}^{P}(\omega)=\frac{\lambda_{\max }\left(\mathbf{B}^{H}(\omega) \hat{\mathbf{R}} \mathbf{B}(\omega)\right)}{p^{2}}
$$

where $\lambda_{\max }(\cdot)$ is the maximum eigenvalue operator. For each frequency $\omega$, the maximum eigenvalue and associated eigenvector of the linear system

$$
\mathbf{B}^{H}(\omega) \hat{\mathbf{R}} \mathbf{B}(\omega) \mathbf{k}_{\max }=\lambda_{\max } \mathbf{k}_{\max }
$$

have to be calculated. The dimension of the Hermitian matrix $\mathbf{B}^{H}(\omega) \hat{\mathbf{R}} \mathbf{B}(\omega) \in \mathbf{M a t}_{N_{\mathrm{pol}}}(\mathbb{C})$ does not depend on the number of antennas $p$. The unitary polarimetric scattering type $\mathbf{k}_{\max }$ permits the extraction of the physical behavior of the reflector. The polarization combination represented by $\mathbf{k}_{\max }$ is optimal with respect to the beamforming criterion. The frequency estimates $\hat{\boldsymbol{\omega}}=\left[\hat{\omega}_{1}, \ldots, \hat{\omega}_{N_{s}}\right]^{\mathrm{T}}$ are given by the locations of the $N_{s}$ maxima of the spectrum $\hat{P}_{\mathrm{BF}}^{P}$. The polarimetric reflectivity at frequency $\hat{\omega}_{i}$ is estimated by $\hat{\tau}_{i}=\hat{P}_{\mathrm{BF}}^{P}\left(\hat{\omega}_{i}\right)$.

\section{Polarimetric Capon Method}

The Capon technique has been proposed for sensors possessing polarization diversity on receive in [43]. The polarimetric Capon algorithm to process polarimetric MB InSAR observations is devised in the context of designing a filter. The singlepolarization MB InSAR steering vector $\mathbf{a}(\omega)$ is replaced by the MBPI steering vector $\mathbf{b}(\omega, \mathbf{k})$. The minimization problem of the polarimetric Capon approach can be expressed as

$$
\min _{\mathbf{h}} \mathbf{h}^{H} \mathbf{R} \mathbf{h} \quad \text { subject to } \quad \mathbf{h}^{H} \mathbf{b}(\omega, \mathbf{k})=1 .
$$

The derivation of the polarimetric Capon filter employing the quadratic minimization theorem results in

$$
\mathbf{h}_{C}^{P}=\frac{\mathbf{R}^{-1} \mathbf{b}(\omega, \mathbf{k})}{\mathbf{b}^{H}(\omega, \mathbf{k}) \mathbf{R}^{-1} \mathbf{b}(\omega, \mathbf{k})} .
$$

The power of the filtered signal is given by

$$
E\left\{\left|y_{F}(l)\right|^{2}\right\}=E\left\{\left|\left(\mathbf{h}_{C}^{P}\right)^{H} \mathbf{y}(l)\right|^{2}\right\}=\frac{1}{\mathbf{b}^{H}(\omega, \mathbf{k}) \mathbf{R}^{-1} \mathbf{b}(\omega, \mathbf{k})} .
$$

It can be maximized in terms of the polarization state $\mathrm{k}$ by

$$
\max _{\|\mathbf{k}\|_{2}=1} \frac{1}{\mathbf{b}^{H}(\omega, \mathbf{k}) \mathbf{R}^{-1} \mathbf{b}(\omega, \mathbf{k})} .
$$

This maximization problem can be solved by computing the minimum eigenvalue of the Hermitian matrix $\mathbf{B}^{H}(\omega) \mathbf{R}^{-1} \mathbf{B}(\omega) \in \mathbf{M a t}_{N_{\mathrm{pol}}}(\mathbb{C})$

$$
\mathbf{B}^{H}(\omega) \mathbf{R}^{-1} \mathbf{B}(\omega) \mathbf{k}_{\min }=\lambda_{\min } \mathbf{k}_{\min } .
$$

Substituting the data covariance matrix $\mathbf{R}$ by the sample covariance matrix $\hat{\mathbf{R}}$, the spectrum of the polarimetric Capon is

$$
\hat{P}_{C}^{P}(\omega)=\frac{1}{\lambda_{\min }\left(\mathbf{B}^{H}(\omega) \hat{\mathbf{R}}^{-1} \mathbf{B}(\omega)\right)}
$$

where $\lambda_{\min }(\cdot)$ is the minimum eigenvalue operator. This means that, for each frequency $\omega$, the minimal eigenvalue and its corresponding eigenvector of the linear system

$$
\mathbf{B}^{H}(\omega) \hat{\mathbf{R}}^{-1} \mathbf{B}(\omega) \mathbf{k}_{\min }=\lambda_{\min } \mathbf{k}_{\min }
$$

have to be computed. If $L \geq p N_{\mathrm{pol}}$ and the noise term has a positive definite covariance matrix, the inverse matrix $\hat{\mathbf{R}}^{-1}$ exists. Compared with the single-polarization Capon method, this implies more averaging and leads to reduced azimuth-range resolution. The dimension of the Hermitian matrix $\mathbf{B}^{H}(\omega) \hat{\mathbf{R}}^{-1} \mathbf{B}(\omega) \in \mathbf{M a t}_{N_{\text {pol }}}(\mathbb{C})$ only depends on the polarization number $N_{\mathrm{pol}}$ and is not a function of the number of 
antennas $p$. The minimum eigenvector $\mathbf{k}_{\min },\left\|\mathbf{k}_{\min }\right\|_{2}=1$, can be regarded as a polarimetric reflection mechanism and allows for determining the physical features of the target. It specifies the optimum polarization combination with respect to the polarimetric Capon algorithm. The frequency estimates $\hat{\boldsymbol{\omega}}=$ $\left[\hat{\omega}_{1}, \ldots, \hat{\omega}_{N_{s}}\right]^{\mathrm{T}}$ correspond to the locations of the $N_{s}$ highest peaks of the spectrum $\hat{P}_{C}^{P}(\omega)$. The polarimetric reflectivity at frequency $\hat{\omega}_{i}$ is extracted by $\hat{\tau}_{i}=\hat{P}_{C}^{P}\left(\hat{\omega}_{i}\right)$.

\section{Polarimetric MUSIC Algorithm}

The MUSIC algorithm has been extended to the particular scenario of polarization diversity in the receive channel in [29] and [43]. Conforming to the development of the conventional MUSIC method, the polarimetric MUSIC technique to process polarimetric MB InSAR measurements is derived.

The MBPI steering vectors $\mathbf{b}\left(\omega_{i}, \mathbf{k}_{i}\right), i=1, \ldots, N_{s}$, are assumed to be linearly independent $\left(N_{s}<\tilde{p}\right)$. Let $\lambda_{1} \geq \cdots \geq \lambda_{\tilde{p}}$ be the eigenvalues of the covariance matrix $\mathbf{R}$ in a nonincreasing order. The orthonormal eigenvectors $\left(\mathbf{f}_{1}, \ldots, \mathbf{f}_{N_{s}}\right)$ are associated with $\left(\lambda_{1}, \ldots, \lambda_{N_{s}}\right)$, and the orthonormal eigenvectors $\left(\mathbf{g}_{1}, \ldots, \mathbf{g}_{\tilde{p}-N_{s}}\right)$ correspond to $\left(\lambda_{N_{s}+1}, \ldots, \lambda_{\tilde{p}}\right)$. The matrices $\mathbf{F}=\left[\mathbf{f}_{1}, \ldots, \mathbf{f}_{N_{s}}\right] \in \operatorname{Mat}_{\tilde{p}, N_{s}}(\mathbb{C})$ and $\mathbf{G}=\left[\mathbf{g}_{1}, \ldots, \mathbf{g}_{\tilde{p}-N_{s}}\right] \in$ $\operatorname{Mat}_{\tilde{p}, \tilde{p}-N_{s}}(\mathbb{C})$ of eigenvectors generate the signal and the noise subspace, respectively. The fundamental assertion for developing the polarimetric MUSIC method can be stated in the following way.

The true frequency values $\left\{\omega_{i}\right\}_{i=1}^{N_{s}}$ and their related reflection mechanisms $\left\{\mathbf{k}_{i}\right\}_{i=1}^{N_{s}}$ are the only solutions of

$$
\mathbf{b}^{H}(\omega, \mathbf{k}) \mathbf{G G}^{H} \mathbf{b}(\omega, \mathbf{k})=0 .
$$

By means of the main proposition (33), the polarimetric spectral MUSIC technique is formulated as follows: After calculating the sample covariance matrix $\hat{\mathbf{R}}$, its eigendecomposition provides the eigenvalues $\hat{\lambda}_{1} \geq \cdots \geq \hat{\lambda}_{\tilde{p}}$ and the eigenvectors $\left(\hat{\mathbf{f}}_{1}, \ldots, \hat{\mathbf{f}}_{N_{s}}\right)$ and $\left(\hat{\mathbf{g}}_{1}, \ldots, \hat{\mathbf{g}}_{\tilde{p}-N_{s}}\right)$ that span the signal and noise subspace, respectively. Using the matrices $\hat{\mathbf{F}}=\left[\hat{\mathbf{f}}_{1}, \ldots, \hat{\mathbf{f}}_{N_{s}}\right] \in \operatorname{Mat}_{\tilde{p}, N_{s}}(\mathbb{C})$ and $\hat{\mathbf{G}}=\left[\hat{\mathbf{g}}_{1}, \ldots, \hat{\mathbf{g}}_{\tilde{p}-N_{s}}\right] \in$ $\operatorname{Mat}_{\tilde{p}, \tilde{p}-N_{s}}(\mathbb{C})$, the pseudospectrum of the polarimetric MUSIC algorithm is expressed as

$$
\hat{P}_{M U}^{P}(\omega)=\frac{1}{\lambda_{\min }\left(\mathbf{B}^{H}(\omega) \hat{\mathbf{G}} \hat{\mathbf{G}}^{H} \mathbf{B}(\omega)\right)} .
$$

The minimum eigenvalue and its eigenvector of the linear system

$$
\mathbf{B}^{H}(\omega) \hat{\mathbf{G}} \hat{\mathbf{G}}^{H} \mathbf{B}(\omega) \mathbf{k}_{\min }=\lambda_{\min } \mathbf{k}_{\min }
$$

have to be evaluated for each frequency $\omega$.

If the linear system (35) does not have full rank, then $\lambda_{\min }=$ 0 , the spectrum (34) is infinite, and the spatial frequencies cannot be retrieved. If the matrix in (35) is nonsingular, then

$$
\tilde{p} \geq N_{s}+N_{\text {pol }}
$$

This implies that the dimension of the noise subspace must not be less than $N_{\text {pol }}, \operatorname{rank}(\hat{\mathbf{G}}) \geq N_{\text {pol }}$. Relation (36) is a necessary
TABLE II

Main DuAl-Baseline InSAR DATA ACQuisition PARAMEters

\begin{tabular}{|l|c|c|}
\hline & Near range & Far range \\
\hline Incidence angle [deg] & 25.0 & 54.9 \\
\hline Range to targets [m] & 3741.70 & 5892.10 \\
\hline$B_{\perp}^{s b}[\mathrm{~m}]$ & -8.96 & -5.65 \\
\hline$H_{a m b}^{s b}[\mathrm{~m}]$ & -20.35 & -98.28 \\
\hline$\kappa_{z}^{s b}[1 / \mathrm{m}]$ & -0.3188 & -0.0639 \\
\hline$B_{\perp}^{l b}[\mathrm{~m}]$ & -35.45 & -21.05 \\
\hline$H_{a m b}^{l b}[\mathrm{~m}]$ & -5.15 & -26.39 \\
\hline$\kappa_{z}^{l b}[1 / \mathrm{m}]$ & -1.22115 & -0.238059 \\
\hline
\end{tabular}

condition for the matrix in (35) to have full rank. It is crucial to note that the maximum number of backscattering components whose spatial frequencies can be estimated by the polarimetric MUSIC method is limited by

$$
N_{s} \leq N_{\text {pol }}(p-1) .
$$

It is clear that the single-polarization mode is included in (37) as a special case.

The dimension of the Hermitian matrix $\mathbf{B}^{H}(\omega) \hat{\mathbf{G}} \hat{\mathbf{G}}^{H} \mathbf{B}(\omega) \in \mathbf{M a t}_{N_{\text {pol }}}(\mathbb{C})$ is independent of the number of sensors $p$. The unitary eigenvector $\mathbf{k}_{\min }$ can be interpreted as a polarimetric scattering mechanism allowing a polarimetric analysis to retrieve the physical properties of the reflector. It encodes the polarization combination that is optimal in the sense of MUSIC. The frequency estimates $\hat{\boldsymbol{\omega}}=\left[\hat{\omega}_{1}, \ldots, \hat{\omega}_{N_{s}}\right]^{\mathrm{T}}$ are related to the positions of the $N_{s}$ largest peaks of the pseudospectrum $\hat{P}_{M U}^{P}(\omega)$. The polarimetric pseudoreflectivity at frequency $\hat{\omega}_{i}$ is determined by $\hat{\tau}_{i}=\hat{P}_{M U}^{P}\left(\hat{\omega}_{i}\right)$.

\section{EXPERIMENTAL RESULTS}

To compare the conceived array signal processing techniques and demonstrate their performances, they are applied to a data set consisting of fully polarimetric and repeat-pass interferometric SAR measurements including three tracks (dual baseline) at L-band with 1.3-GHz center frequency. It was acquired by DLR's E-SAR system [44] over the city of Dresden in Germany on August 1, 2000. The main data acquisition parameters of the dual-baseline system comprising a small baseline $(s b)$ of around $10 \mathrm{~m}$ and a large baseline $(l b)$ of about $40 \mathrm{~m}$ are summarized in Table II. It includes the incidence angles $\theta$, ranges to targets $r$, orthogonal baselines $B_{\perp}^{\mathrm{sb}, \mathrm{lb}}$, heights of ambiguity $H_{\mathrm{amb}}^{\mathrm{sb}, \mathrm{lb}}$, and the vertical wavenumbers $\kappa_{z}^{\mathrm{sb}, \mathrm{lb}}$ for near and far ranges. The resolutions of the three POLSAR images are $3.0 \mathrm{~m}$ in azimuth and $2.2 \mathrm{~m}$ in range. Hence, it is reasonable to assume that the Gaussian data model is valid in the examined urban area since each resolution cell contains generally a sufficient number of reflectors [49].

\section{A. Building Layover 3-D Imaging Using Beamforming, Capon, and MUSIC Algorithms}

First, a building layover is studied to compare the singlepolarization and fully polarimetric beamforming, Capon, and MUSIC methods [48]. The reflection processes inside the building layover are shown in Fig 2: Going from far range in the 


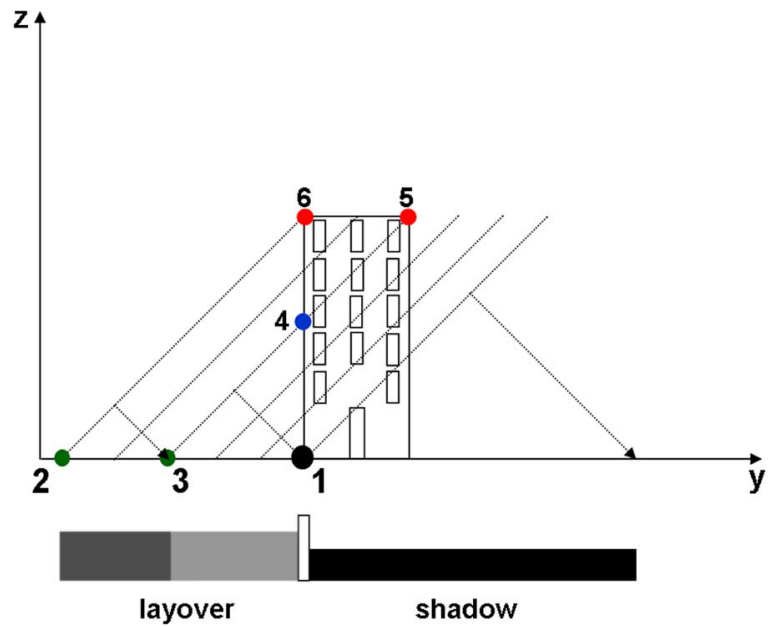

Fig. 2. Schematic view of building layover with sensor on the left-hand side. The wall-ground interaction point in far range is indicated by the number one, the range resolution cell inside the layover that is closest to the sensor position by the number two. At the bottom of the scheme, the (black color) building shadow, (white box) wall-ground interaction point, and (gray colors) layover are shown in ground range [6]. The layover can be split up into one part including backscattering from the (dark gray) roof, ground, and wall and another part comprising reflection from the (light gray) ground and wall, but not from the roof. The dots represent the expected scattering inside the building layover: The reflection at the bottom stems from the (green color) ground and (black color) wall-ground interaction point, (blue color) in the middle from the facade, and (red color) on top from the building roof. The scatterers labeled with the numbers three, four, and five are within one resolution cell, those associated with the numbers two and six within another.
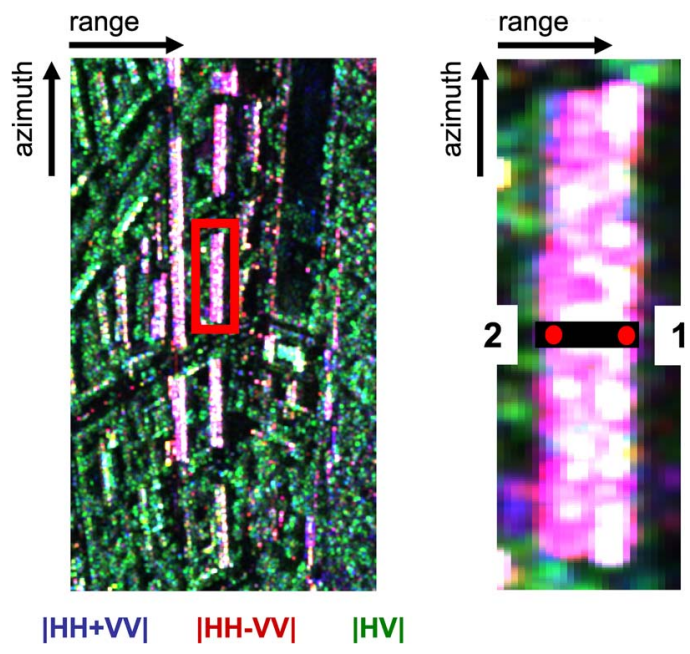

Fig. 3. (Left) Pauli color-coded urban scene. The along-track direction is on the left-hand side, i.e., far range on the right-hand side. (Right) Close-up view of the building layover inside the red box.

direction of the SAR antenna location, i.e., from right to left, the wall-ground interaction point is located at the topographic height. It is represented by the number one. Miscellaneous scattering phenomena emerge in the middle of layover, originating from the ground, wall, and roof. At the pixels of the building layover which are nearest to the sensor, i.e., on the left-hand side at number two, reflection effects arise from the ground and the roof inside one range resolution element.

For inspecting the layover, a building in midrange shown in Fig. 3 is chosen, where the height of ambiguity of the acquisition system is $\left|H_{a m b}^{\mathrm{sb}}\right| \approx 67.5 \mathrm{~m}$ for the small baseline and $\left|H_{\mathrm{amb}}^{\mathrm{lb}}\right| \approx 15 \mathrm{~m}$ for the large baseline. Thus, the height
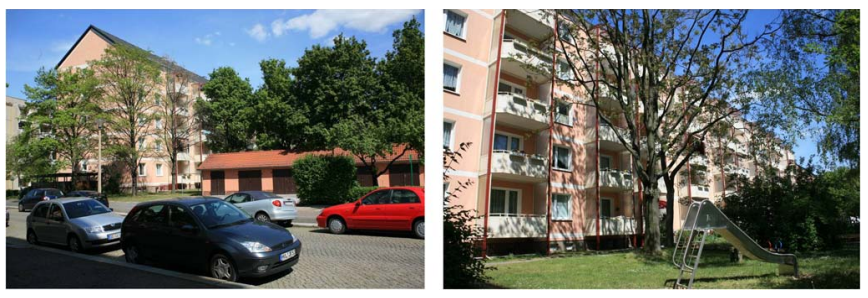

Fig. 4. Optical image of building. (Left) View from the side. (Right) View from the front (copyright G. S.).

Rayleigh resolution [30] corresponds to approximately $15 \mathrm{~m}$. Far range is located on the right and near range on the left-hand side, i.e., the along-track direction is on the left-hand side. This means that the edifice is parallel to the sensor trajectory. As can be appreciated in Fig. 4, it is made up of five floors and a pitched roof, resulting in a height of about $17 \mathrm{~m}$.

Tomographic slices are calculated by the beamforming and Capon methods using observations in $v v$ channel and fully polarimetric measurements. The single-polarization and fully polarimetric MUSIC algorithms generate pseudotomograms. The 3-D images are computed in the range $[-10 \mathrm{~m}, 25 \mathrm{~m}]$ in the vertical direction. ${ }^{1}$ A $5 \times 5$ boxcar filter is employed to spatially average the covariance matrix. Despite the fact that spatial filtering decreases the azimuth-range resolution, it is generally applied for the purpose of reducing the speckle effect [25], [30] and noise, even in urban scenarios [8]. In any case, in current sensor systems, the number of available baselines and, thus, the achievable vertical resolution seem to be the limiting factors rather than the azimuth-range resolution. The (pseudo)tomograms are extracted for the samples along the line inside the building layover shown in Fig. 3 on the right-hand side. The (pseudo)reflectivities are always depicted in the range from 0.0 to 2.5 times the mean of the amplitude in the entire image.

The tomographic slices shown in Fig. 5 are calculated by the single-polarization and fully polarimetric beamforming algorithms, respectively. On the right-hand side, the backscattering from the wall-ground interaction at the topographic height of approximately $0 \mathrm{~m}$ is visible. The main lobe is broad, and the side lobes are rather strong, particularly for the polarimetric beamforming. It can be noted that the reflection from the wall-ground interaction sample is not focused in one range element, as predicted by theoretical considerations [50]. This can be explained by the use of the boxcar filter. In the middle of the 3-D image, the single-polarization beamformer shows reflectors between 10 and $15 \mathrm{~m}$ associated with the building wall. The high side lobes below $0 \mathrm{~m}$ are caused by the irregular sampling. Compared to and due to the very bright backscattering from the wall-ground interaction point [8], [50], the reflectivity is weak at the left-hand side for the singlepolarization and fully polarimetric and in the middle for the fully polarimetric beamforming method.

The 3-D imaging results of the single-polarization and fully polarimetric Capon techniques are shown in Fig. 6. On the right side, both algorithms detect a signal at around $0 \mathrm{~m}$, originating

\footnotetext{
${ }^{1}$ As the vertical wavenumber is normalized by $1 / \sin \theta$ [see its definition after (4)], the vertical axis of the 3-D images is related to the vertical direction and not to the direction perpendicular to the line of sight.
} 

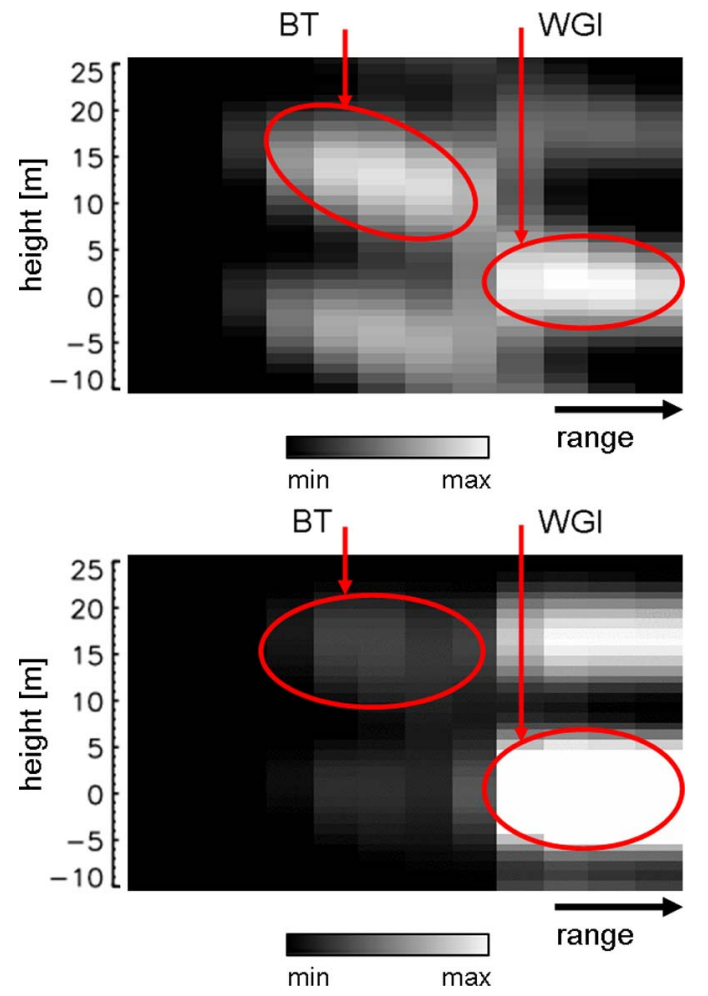

Fig. 5. Three-dimensional imaging of building layover. The horizontal axis corresponds to slant range, with far range on the right-hand side. The vertical axis is related to the estimated height in the vertical direction going from -10 to $25 \mathrm{~m}$. (WGI) Wall-ground interaction. (BT) Building top. (Top) singlepolarization and (bottom) fully polarimetric beamforming tomographic slices.

from the building base. In comparison with the beamforming method, the Capon algorithms lead to finer resolution and better leakage reduction. Going to the left, the elevation of the scatterer increases to about $10 \mathrm{~m}$ for the single-polarization Capon, whereas the fully polarimetric Capon separates two contributions, the first slightly above $0 \mathrm{~m}$ and the second at approximately $15 \mathrm{~m}$ related to the building height. To a limited extent, the fully polarimetric Capon technique possesses sufficiently narrow peaks to identify two components at different height locations. On the account of the large dynamic range [8] caused by the dihedral-wall-ground-corner reflector, the backscattering is very weak on the left-hand side.

The pseudotomograms of the single-polarization and fully polarimetric MUSIC methods with fixed model order one (MO 1) are shown in Fig. 7. Both algorithms are appropriate to recognize one reflector at around $0 \mathrm{~m}$, corresponding to the wall-ground interaction points at the right-hand side. As for beamforming and Capon, it is conspicuous that the dihedralwall-ground scatterer is not compressed in one single range pixel but stretches over several samples due to spatial averaging. Compared to the beamforming and Capon techniques, the spectral peaks of MUSIC with MO 1 are very narrow, and the side lobes are visibly decreased. While the height of the main scatterer rises from around 11 to $18 \mathrm{~m}$ in the single-polarization MUSIC image, the elevation of the main reflector of the fully polarimetric MUSIC method is very stable at approximately $17 \mathrm{~m}$. They are associated with the building wall and roof. The second peaks at around $-2 \mathrm{~m}$ (single-polarization) and $0 \mathrm{~m}$ (fully polarimetric) seem to be side lobes in the height of ambiguity.

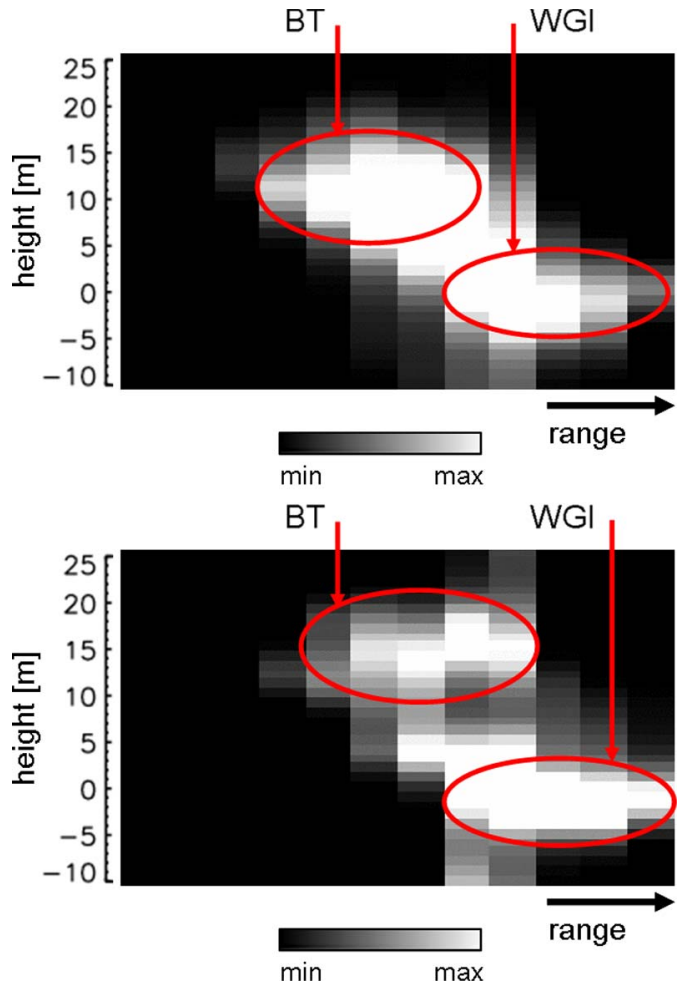

Fig. 6. (Top) single-polarization and (bottom) fully polarimetric Capon tomographic slices.
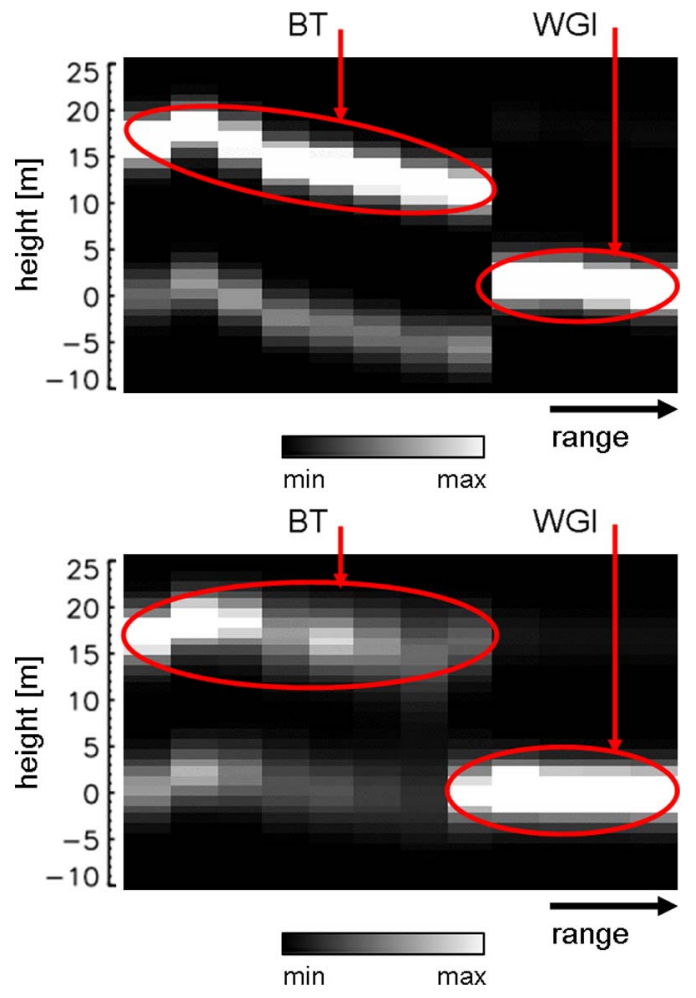

Fig. 7. (Top) single-polarization and (bottom) fully polarimetric MUSIC pseudotomographic slices; model order fixed to one.

The pseudotomographic slices of the single-polarization and fully polarimetric MUSIC methods (see Fig. 8), where the model order is set to two, are quite different: The elevation of one single reflector grows from $-5 \mathrm{~m}$ to more than $10 \mathrm{~m}$ in 


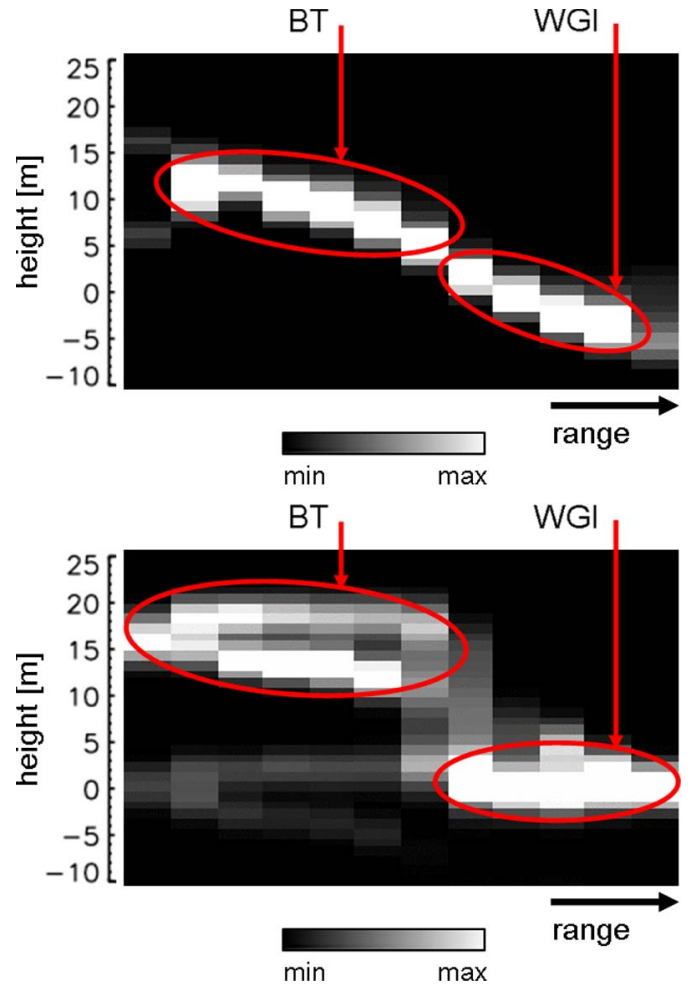

Fig. 8. (Top) single-polarization and (bottom) fully polarimetric MUSIC pseudotomographic slices; model order fixed to two.

the single-polarization MUSIC 3-D image. It has to be noted that the classical MUSIC algorithm of model order two (MO 2) represents a limiting case if it is applied to single-polarization dual-baseline InSAR measurements (see Section II-D). It seems to compute an average phase. On the right-hand side of the fully polarimetric MUSIC pseudotomogram, one source at around $0 \mathrm{~m}$ related to the building base can be seen. Compared to the polarimetric MUSIC algorithm of MO 1, the resolution is slightly deteriorated: This degradation may be explained by overmodeling or an object near the wall-ground interaction points. In the middle and on the left side, two strong scatterers that are very close to each other are distinguished: The first at approximately $13 \mathrm{~m}$ is generated by targets along the building wall, and the second is very stable at $18 \mathrm{~m}$ and caused by the building roof. Side lobes are visible at about $0 \mathrm{~m}$. For the polarimetric MUSIC of model order higher than two [cf. (37)], the imaging quality (not depicted here) worsens due to defocusing and the introduction of additional ambiguities.

The generation of 3-D images from polarimetric dualbaseline interferometric SAR observations can be summarized as follows: The single-polarization and fully polarimetric beamforming methods produce tomographic slices with low resolution and strong side lobes. Even though the single-polarization and fully polarimetric Capon algorithms refine the spectral peaks and reduce the leakage problem, it is still difficult to analyze the tomograms of the building layover. The singlepolarization MUSIC technique of MO 2 estimates an average phase. The 3-D images computed by the single-polarization and fully polarimetric MUSIC algorithms with model order set to one show very high resolution and considerably diminished leakage: In far range, contributions associated with the

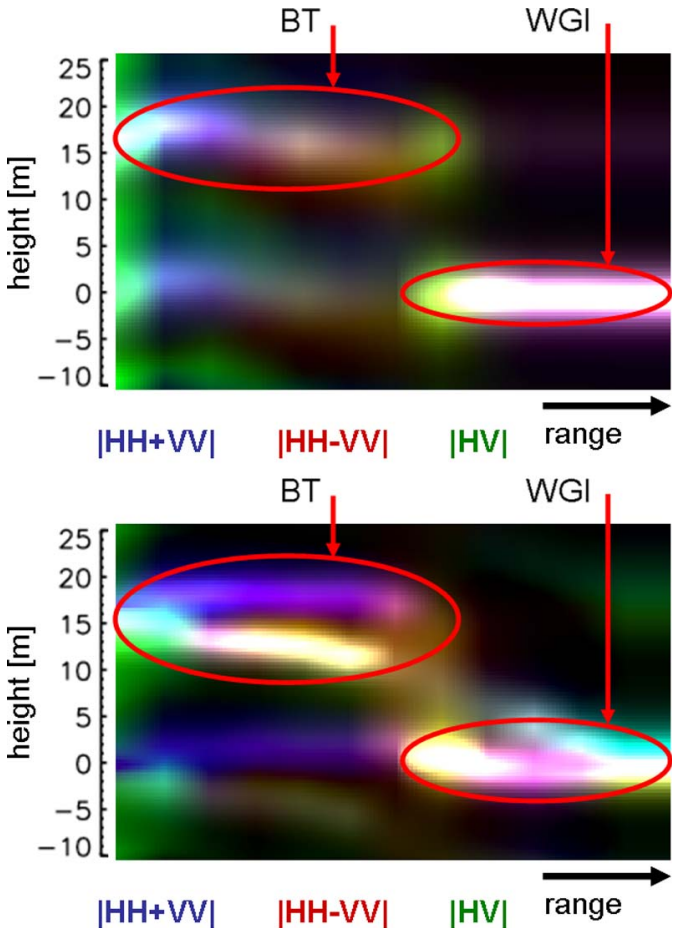

Fig. 9. Pseudotomographic slices of the optimal MUSIC scattering mechanisms $\mathbf{k}_{\min }$ : (Top) Model order fixed to one and (bottom) model order fixed to two.

wall-ground interaction points are clearly detected. In near range, the reflectors are related to the building wall and top. The pseudotomographic slice of the polarimetric MUSIC technique whose model order is fixed to two exhibits one signal at the building base in far range. Compared to the MUSIC methods of order one, the spectral pattern is broadened due to overmodeling or a target near the ground-wall interaction points. For the samples closer to the antenna positions, the polarimetric MUSIC of MO 2 is capable of identifying two neighboring objects within one azimuth-range resolution cell: The first scatterer is located at the building roof, and the second is along the wall. The height separation is much lower than the height of ambiguity of the large baseline, i.e., far beyond the Rayleigh resolution. Single-polarization and fully polarimetric MUSICs have been clearly identified as the algorithms having the best estimation accuracy in terms of spatial resolution, leakage reduction, and target separation. Thus, the following investigations are focused on the MUSIC method.

\section{B. Building Layover Scattering Mechanism Estimation Using Polarimetric MUSIC Method}

Next, the optimal MUSIC scattering vectors $\mathbf{k}_{\min }$ are employed to form 3-D images illustrating the reflection processes [40], [51]. Fig. 9 shows the scattering mechanisms computed by the fully polarimetric MUSIC method with model orders set to one and two, respectively. On the right-hand side, the relative powers between the polarizations are alike. For the polarimetric MUSIC algorithm with model order fixed to one, the first component of the Pauli polarization basis associated with surface scattering prevails on the left side at the building top. The MUSIC method of MO 2 in Fig. 9 (bottom) reveals 

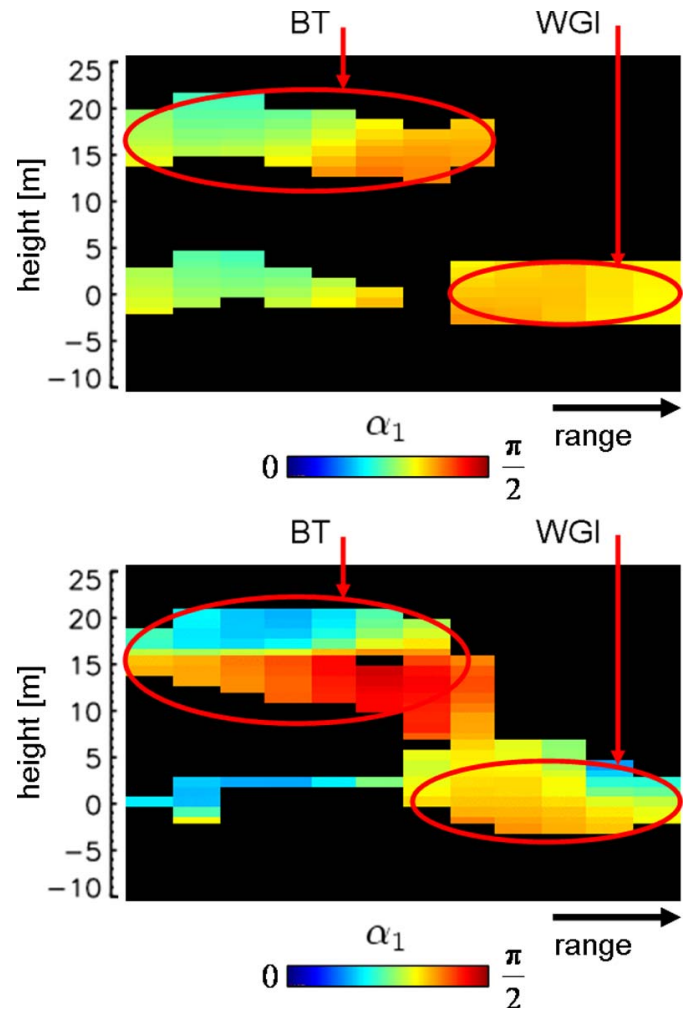

Fig. 10. Polarimetric indicator $\alpha_{1}$ of the optimal MUSIC scattering vector: (Top) Model order fixed to one and (bottom) model order fixed to two.

surface reflection on the building roof (the bluish stripe inside the red ellipse on the left-hand side) and an additional scattering phenomenon very close to the building top where the polarization channels have quite equal amplitudes. It is evident that these reflection processes, which are only separated by a few meters, exhibit very disparate polarization signatures.

In the following, scattering patterns are examined in more details by using the polarimetric indicator $\alpha_{1}$ of the dominant reflector inside the optimal vectors $\mathbf{k}_{\text {min }}$. In Fig. 10 (top: MO 1; bottom: MO 2), a mask based on a threshold of the pseudoreflectivities is applied to set the samples to black color, whose backscattering power is less than the mean of the imaged area. On the right-hand side at the wall-ground interaction points, the $\alpha_{1}$ value is rather high, suggesting double-bounce reflection. In the middle part of the image, the polarimetric descriptor reaches values of about $\pi / 2$, linked to the doublebounce class. At the left-hand side, $\alpha_{1}$ attains average to low values at the roof that are related to surface reflection. Again, it is conspicuous that the polarimetric MUSIC algorithm of MO 2 is adequate to differentiate, within one azimuth-range resolution cell, two neighboring scatterers that possess very distinct polarimetric behavior: Whereas, at the roof, the $\alpha_{1}$ value is low and corresponds to surface scattering, it is high for the sources below the building top and related to double-bounce reflection.

\section{Large-Scene Analysis Using Single-Polarization and Fully Polarimetric MUSIC Methods}

So far, the layover of one single building has been investigated. Now, 3-D images of a large scene are generated along the

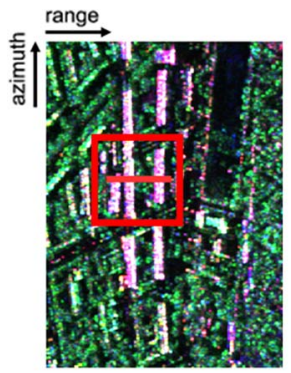

$|H H+V V||H H-V V| \quad|H V|$

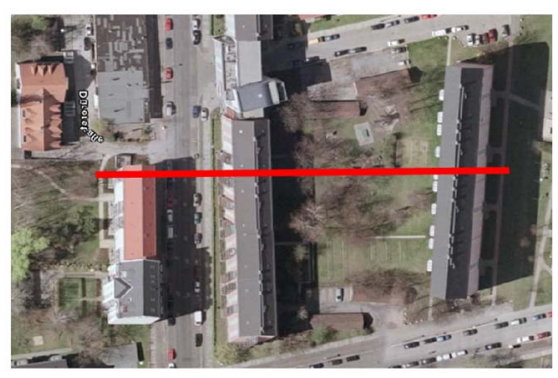

Fig. 11. Large scene containing three tall buildings and a spacious courtyard with trees. (Left) POLSAR image with azimuth on the left-hand side. The sample line is marked by red color. (Right) Optical image (copyright Google Earth) of the area enclosed by the red box in the POLSAR image.

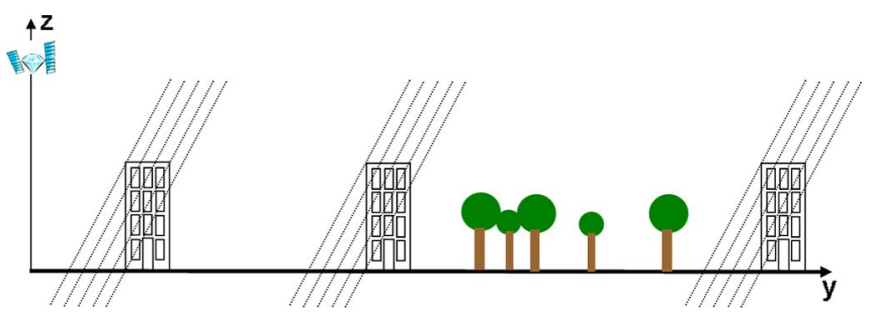

Fig. 12. Diagram of the large scene containing three tall buildings and a spacious courtyard with trees.
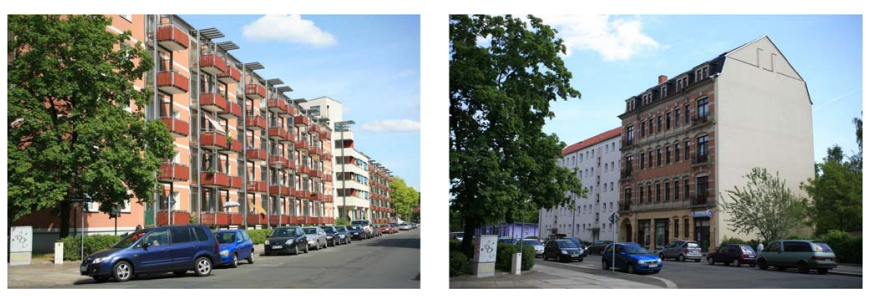

Fig. 13. Optical image. (Left) Building number two. (Right) Building number three (copyright G.S.).

sample line defined in Fig. 11 (left). The area contains three tall buildings, as shown in Fig. 12. Building numbers one and two on the right enclose a spacious courtyard shown in Figs. 4 and 11 (right), consisting of several trees, small barracks, hanging bars, and a playground with a slide. Building numbers two and three on the left are separated by a street, as can be seen in Fig. 13. The sensor is located on the left-hand side.

The pseudotomograms are computed by the singlepolarization MUSIC (MO 1) and the fully polarimetric MUSIC (MO 1 and MO 2) algorithms shown in Fig. 14. Going from right to left, i.e., from far to near range, the wall-ground interaction samples of the first building are apparent at about $0 \mathrm{~m}$, followed by the strong backscattering from the roof. In the courtyard, there is one contribution at the topographic height and strong reflectors at approximately $10 \mathrm{~m}$. Some spurious side lobes lie beneath $0 \mathrm{~m}$. In the center of the images is the shadow of building number two, where the MUSIC pseudoreflectivities do not vanish: This phenomenon may be generated by an object like a tree inside the building shadow or it may be caused by MUSIC overmodeling. At the left end of the pseudotomographic slices, two buildings can be recovered: First, signals at around $0 \mathrm{~m}$ related to the wall-ground doublebounce reflections appear, then a component located at the roof 

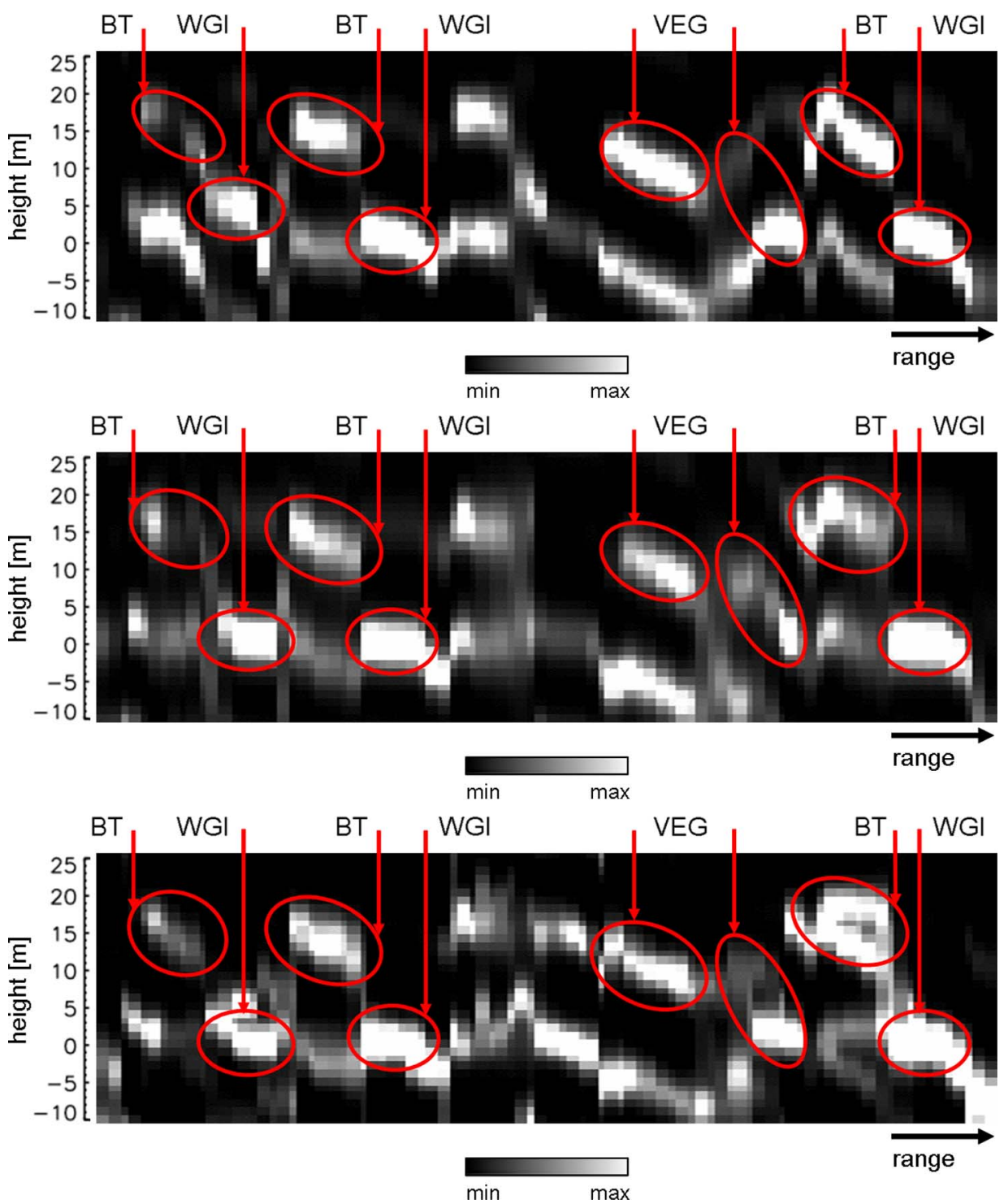

Fig. 14. Large scene containing three buildings and a spacious courtyard. The following acronyms are used: WGI-wall-ground interaction, BT—building top, and VEG-vegetation. (Top) single-polarization MUSIC pseudotomographic slice, model order fixed to one; (middle) fully polarimetric MUSIC pseudotomographic slice, model order fixed to one; and (bottom) fully polarimetric MUSIC pseudotomographic slice, model order fixed to two.

is detected. Globally, the polarimetric MUSIC with MO 1 3-D image can be interpreted rather easily, whereas the scattering processes of the single-polarization MUSIC MO 1 and fully polarimetric MUSIC with MO 2 methods are more difficult to analyze.

The interpretation of the reflection pattern is facilitated by the optimal MUSIC scattering vectors $\mathbf{k}_{\min }$ : Fig. 15 shows the reflection mechanisms of the fully polarimetric MUSIC technique with model order fixed to one and two, respectively. The wall-ground interaction points of the three buildings are associated with the second component of the Pauli polarization basis $(h h-v v)$. At the roofs, the first $(h h+v v)$ and second contributions are dominant for buildings one and two. Volume reflection $(h v)$ can be observed at the top of building three, which may be explained by its structure or its orientation with respect to the flight path. Inside the courtyard, the backscattering of an object at around $10 \mathrm{~m}$ is related to the third term of the Pauli basis. The rather broad peak and the high side lobes below $0 \mathrm{~m}$ suggest that it represents the response of a distributed target such as a tree canopy. The strong signal at approximately $0 \mathrm{~m}$ on its right side might be caused by backscattering from the tree trunk and ground.

\section{Discussion AND CONCLUSION}

In this paper, new polarimetric algorithms have been conceived to estimate the polarimetric reflectivities and reflection types from MB POL-InSAR data for the purpose of 3-D imaging. First, a signal model has been adapted to the polarimetric case, leading to the concepts of the MBPI steering vector and the polarimetric reflectivity. Subsequently, the spectral analysis techniques have been generalized to the polarimetric MB InSAR configuration. These new methods enhance the phase estimation of scatterers not only by increasing the dimension of the observation space but also via calculating optimal polarization combinations. They permit the retrieval 

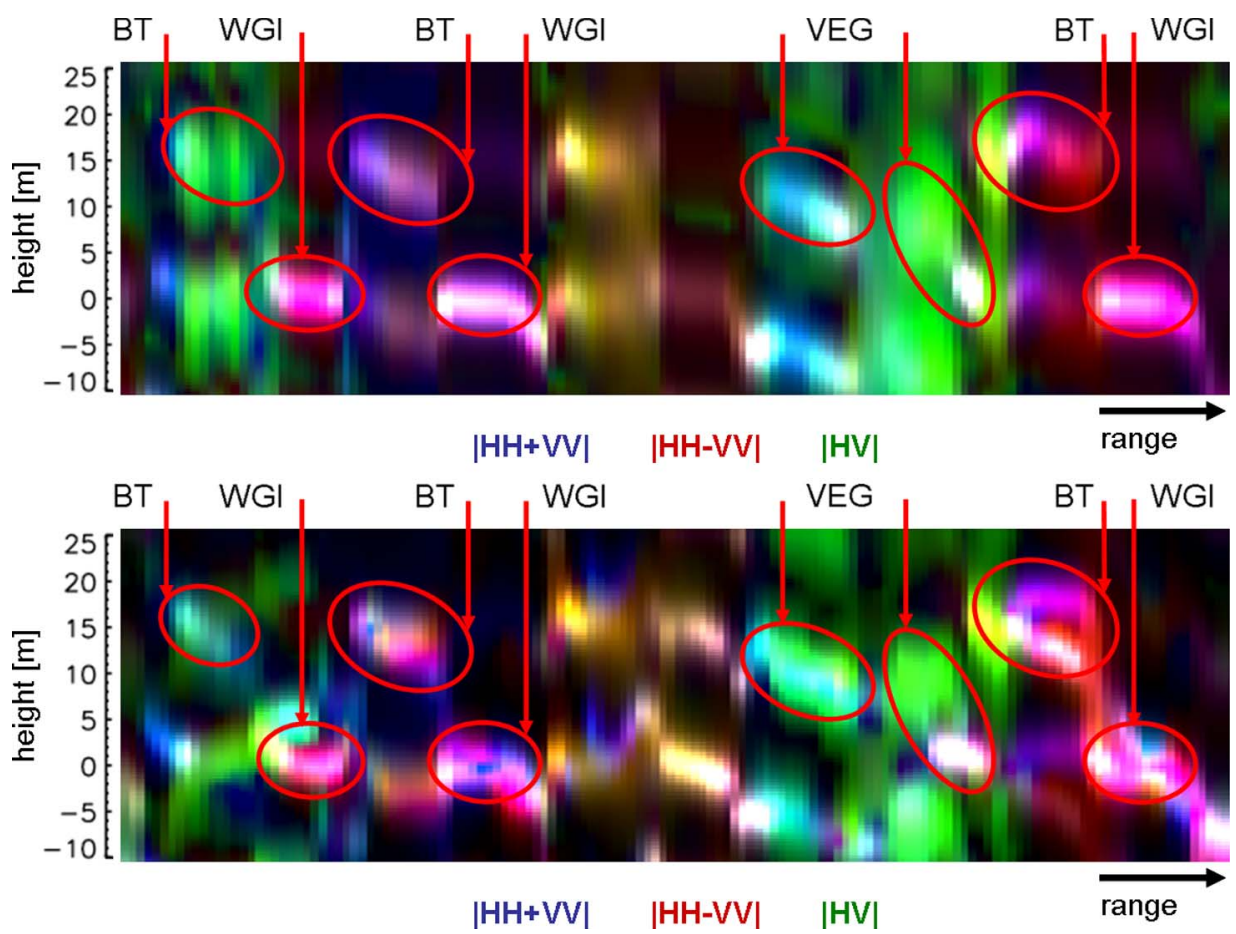

Fig. 15. Large scene: Pseudotomographic slice of the optimal MUSIC scattering mechanisms $\mathbf{k}_{\mathrm{min}}$. (Top) Model order fixed to one. (Bottom) Model order fixed to two.

of the spatial frequencies, the associated optimal scattering vectors, and the polarimetric (pseudo)reflectivities. The optimal scattering mechanisms allow for examining the reflector physical properties by analyzing their polarimetric behavior. The nonparametric beamforming and Capon algorithms have been generalized to the MB POL-InSAR scenario to extract the spatial frequencies, the corresponding optimal reflection mechanisms, and the polarimetric reflectivities. The superresolution polarimetric MUSIC approach has been conceived to determine the spatial frequencies, the related scattering types, and the polarimetric pseudoreflectivities. Their mathematical features have been explained in detail.

The proposed techniques have been applied to fully polarimetric dual-baseline interferometric SAR measurements of an urban scene to compare and validate their performances. First, the layover of a single building has been thoroughly examined by means of 3-D imaging: The single-polarization and fully polarimetric beamforming algorithms generate 3-D images having broad peaks and significant leakage. Although the single-polarization and polarimetric Capon methods are capable of narrowing the resolution and diminishing the side lobes, they remain intricate to recognize the complex characteristics of the building layover. The single-polarization MUSIC technique of MO 2 detects only the height of one source.

The pseudotomographic slices calculated by the singlepolarization and fully polarimetric MUSIC algorithms of MO 1 exhibit fine resolution and noticeably reduced side lobes: For the wall-ground interaction samples in far range, one backscattering source at the building base is identified. The elevation of one component can be determined inside one azimuth-range resolution cell close to the sensor position, corresponding to the roof. The 3-D image of the polarimetric MUSIC method of
MO 2 shows one target in far range at the ground. Overmodeling or an object near the ground-wall interaction points blurs slightly the peak in comparison with the polarimetric MUSIC approach of MO 1. The polarimetric MUSIC MO 2 algorithm discerns two neighboring constituents within a single azimuth-range resolution cell for samples close to the sensor location: One reflector is positioned on the building top, and the other is along the wall. The height separation is far beyond the Rayleigh resolution, i.e., much lower than the height of ambiguity of the large baseline.

The MUSIC methods, particularly the polarimetric MUSIC, possess the best performance among the spectral estimation techniques regarding spatial resolution, side lobe suppression, and target identification. They are most robust to process data acquired by a nonuniform nonlinear sensor array. These techniques are appropriate for solving the building layover problem, i.e., recovering two or more components within one azimuth-range resolution cell.

Examining the optimal polarimetric MUSIC reflection types supports the 3-D image interpretation: Double bounce prevails at the wall-ground interaction points. The scatterers caused by the roof are related to surface reflection. In near range, the polarimetric MUSIC technique of MO 2 differentiates two close sources associated with disparate polarization signatures: Besides surface scattering from the building top, the targets along the wall correspond to double bounce.

Finally, large heterogeneous urban areas have been analyzed, including several buildings, other man-made objects, and different kinds of vegetation. The building base and roof are conspicuous. Moreover, the heights of reflection effects induced by natural distributed targets have been determined. 
In the future, the developed techniques shall be applied to tomographic observations including more than two baselines. This will lead to enhanced 3-D imaging quality with respect to refined resolution in elevation and leakage reduction. Furthermore, the performances of fully polarimetric and dualpolarization approaches shall be compared: It is anticipated that copolarized channels $(h h+v v$ and $h h-v v)$ are most important in urban areas, whereas copolarized and cross-polarized measurements (e.g., $v v$ and $h v$ ) are essential for forested scenes. These studies can be carried out using airborne data sets, e.g., from the E-SAR/F-SAR system of DLR, or spaceborne measurements, e.g., from TerraSAR-X and TanDEM-X.

\section{ACKNOWLEDGMENT}

The authors would like to thank the anonymous reviewers for their valuable comments and suggestions that helped to improve the readability of this paper. The first author would like to express his gratitude to G. and G. Sauer for providing the optical building images.

\section{REFERENCES}

[1] T. Macrì Pellizzeri, P. Gamba, P. Lombardo, and F. Dell'Acqua, "Multitemporal/multiband SAR classification of urban areas using spatial analysis: Statistical versus neural kernel-based approach," IEEE Trans. Geosci. Remote Sens., vol. 41, no. 10, pp. 2338-2353, Oct. 2003.

[2] R. Zandoná Schneider, K. P. Papathanassiou, I. Hajnsek, and A. Moreira, "Polarimetric and interferometric characterization of coherent scatterers in urban areas," IEEE Trans. Geosci. Remote Sens., vol. 44, no. 4, pp. 971984, Apr. 2006.

[3] D. Perissin and F. Rocca, "High-accuracy urban DEM using permanent scatterers," IEEE Trans. Geosci. Remote Sens., vol. 44, no. 11, pp. 33383347, Nov. 2006.

[4] C. Tison, F. Tupin, and H. Maître, "A fusion scheme for joint retrieval of urban height map and classification from high-resolution interferometric SAR images," IEEE Trans. Geosci. Remote Sens., vol. 45, no. 2, pp. 496505, Feb. 2007.

[5] K. Iribe and M. Sato, "Analysis of polarization orientation angle shifts by artificial structures," IEEE Trans. Geosci. Remote Sens., vol. 45, no. 11, pp. 3417-3425, Nov. 2007.

[6] A. Thiele, E. Cadario, K. Schulz, U. Thönnessen, and U. Soergel, "Building recognition from multi-aspect high-resolution InSAR data in urban areas," IEEE Trans. Geosci. Remote Sens., vol. 45, no. 11, pp. 3583-3593, Nov. 2007

[7] D. Perissin and A. Ferretti, "Urban-target recognition by means of repeated spaceborne SAR images," IEEE Trans. Geosci. Remote Sens., vol. 45, no. 12, pp. 4043-4058, Dec. 2007.

[8] A. R. Brenner and L. Roessing, "Radar imaging of urban areas by means of very high-resolution SAR and interferometric SAR," IEEE Trans. Geosci. Remote Sens., vol. 46, no. 10, pp. 2971-2982, Oct. 2008.

[9] G. Ferraiuolo, F. Meglio, V. Pascazio, and G. Schirinzi, "DEM reconstruction accuracy in multichannel SAR interferometry," IEEE Trans. Geosci. Remote Sens., vol. 47, no. 1, pp. 191-201, Jan. 2009.

[10] M. Eineder, N. Adam, R. Bamler, N. Yague-Martinez, and H. Breit, "Spaceborne spotlight SAR interferometry with TerraSAR-X," IEEE Trans. Geosci. Remote Sens., vol. 47, no. 5, pp. 1524-1535, May 2009.

[11] L. Denis, F. Tupin, J. Darbon, and M. Sigelle, "Joint regularization of phase and amplitude of InSAR data: Application to 3-D reconstruction," IEEE Trans. Geosci. Remote Sens., vol. 47, no. 11, pp. 3774-3785, Nov. 2009.

[12] R. Bamler and P. Hartl, "Synthetic aperture radar interferometry," Inverse Problems, vol. 14, no. 4, pp. 1-54, Aug. 1998.

[13] P. A. Rosen, S. Hensley, I. R. Joughin, F. K. Li, S. N. Madsen, E. Rodriguez, and R. M. Goldstein, "Synthetic aperture radar interferometry," Proc. IEEE, vol. 88, no. 3, pp. 333-382, Mar. 2000.

[14] S. R. Cloude and E. Pottier, "A review of target decomposition theorems in radar polarimetry," IEEE Trans. Geosci. Remote Sens., vol. 34, no. 2, pp. 498-518, Mar. 1996.
[15] S. R. Cloude and E. Pottier, "An entropy based classification scheme for land applications of polarimetric SAR," IEEE Trans. Geosci. Remote Sens., vol. 35, no. 1, pp. 68-78, Jan. 1997.

[16] A. Freeman and S. L. Durden, "A three-component scattering model for polarimetric SAR data," IEEE Trans. Geosci. Remote Sens., vol. 36, no. 3, pp. 963-973, May 1998.

[17] S. R. Cloude and K. P. Papathanassiou, "Polarimetric SAR interferometry," IEEE Trans. Geosci. Remote Sens., vol. 36, no. 5, pp. 1551-1565, Sep. 1998.

[18] K. P. Papathanassiou and S. R. Cloude, "Single-baseline polarimetric SAR interferometry," IEEE Trans. Geosci. Remote Sens., vol. 39, no. 11, pp. 2352-2363, Nov. 2001.

[19] S. R. Cloude and K. P. Papathanassiou, "Three stage inversion process for polarimetric SAR interferometry," IEEE Proc.-Radar Sonar Navig., vol. 150, no. 3, pp. 125-134, Jun. 2003.

[20] S. R. Cloude, "Polarization coherence tomography," Radio Sci., vol. 41, p. RS4 017, Aug. 2006.

[21] S. R. Cloude, "Dual-baseline coherence tomography," IEEE Geosci. Remote Sens. Lett., vol. 4, no. 1, pp. 127-131, Jan. 2007.

[22] A. Reigber and A. Moreira, "First demonstration of airborne SAR tomography using multibaseline L-band data," IEEE Trans. Geosci. Remote Sens., vol. 38, no. 5, pp. 2142-2152, Sep. 2000.

[23] A. Reigber, "Airborne polarimetric SAR tomography," Ph.D. dissertation, University of Stuttgart, Stuttgart, Germany, Oct. 2001.

[24] F. Gini, F. Lombardini, and M. Montanari, "Layover solution in multibaseline SAR interferometry," IEEE Trans. Aerosp. Electron. Syst., vol. 38, no. 4, pp. 1344-1356, Oct. 2002.

[25] F. Lombardini, M. Montanari, and F. Gini, "Reflectivity estimation for multibaseline interferometric radar imaging of layover extended sources," IEEE Trans. Signal Process., vol. 51, no. 6, pp. 1508-1519, Jun. 2003.

[26] F. Gini and F. Lombardini, "Multibaseline cross-track SAR interferometry: A signal processing perspective," IEEE Aerosp. Electron. Syst. Mag., vol. 20, no. 8, pp. 71-93, Aug. 2005.

[27] J. Homer, I. D. Longstaff, and G. Callaghan, "High resolution 3-D SAR via multi-baseline interferometry," in Proc. IGARSS, Lincoln, May 1996, pp. 796-798.

[28] J. Capon, "High-resolution frequency-wavenumber spectrum analysis," Proc. IEEE, vol. 57, no. 8, pp. 1408-1418, Aug. 1969.

[29] R. O. Schmidt, "Multiple emitter location and signal parameter estimation," in Proc. RADC Estimation Workshop, Oct. 1979, pp. 243-258.

[30] F. Lombardini, L. Rössing, J. Ender, and F. Viviani, "Towards a complete processing chain of multibaseline airborne InSAR data for layover scatterers separation," in Proc. URBAN, Paris, France, Apr. 2007, pp. 1-6.

[31] L. Roessing and J. H. G. Ender, "Multi-antenna SAR tomography using superresolution techniques," in Proc. EUSAR, Munich, Germany, May 2000, pp. 55-58.

[32] F. Lombardini and A. Reigber, "Adaptive spectral estimation for multibaseline SAR tomography with airborne L-band data," in Proc. IGARSS, Toulouse, France, Jul. 2003, pp. 2014-2016.

[33] S. Guillaso and A. Reigber, "Scatterer characterisation using polarimetric SAR tomography," in Proc. IGARSS, Seoul, South Korea, Jul. 2005, pp. $2685-2688$.

[34] S. Guillaso, M. Jäger, and A. Reigber, "Information extraction from tomographic SAR data," in Proc. EUSAR, Dresden, Germany, May 2006.

[35] M. Nannini, R. Scheiber, and A. Moreira, "Estimation of the minimum number of tracks for SAR tomography," IEEE Trans. Geosci. Remote Sens., vol. 47, no. 2, pp. 531-543, Feb. 2009.

[36] H. Yamada, Y. Yamaguchi, Y. Kim, E. Rodriguez, and W.-M. Boerner, "Polarimetric SAR interferometry for forest analysis based on the ESPRIT algorithm," IEICE Trans. Electron., vol. E84-C, no. 12, pp. 1917-1924, Dec. 2001.

[37] S. Guillaso, L. Ferro-Famil, A. Reigber, and E. Pottier, "Building characterisation using L-band polarimetric interferometric SAR data," IEEE Geosci. Remote Sens. Lett., vol. 2, no. 3, pp. 347-351, Jul. 2005.

[38] G. Fornaro, D. Reale, and F. Serafino, "Four-dimensional SAR imaging for height estimation and monitoring of single and double scatterers," IEEE Trans. Geosci. Remote Sens., vol. 47, no. 1, pp. 224-237, Jan. 2009.

[39] X. X. Zhu, N. Adam, and R. Bamler, "Space-borne high resolution tomographic interferometry," in Proc. IGARSS, Cape Town, South Africa, Jul. 2009, pp. IV-869-IV-872.

[40] S. Sauer, L. Ferro-Famil, A. Reigber, and E. Pottier, "Multibaseline POLInSAR analysis of urban scenes for 3-D modeling and physical feature retrieval at L-band," in Proc. IGARSS, Barcelona, Spain, Jul. 2007, pp. 1098-1101. 
[41] S. Sauer, L. Ferro-Famil, A. Reigber, and E. Pottier, "3D urban remote sensing using dual-baseline POL-InSAR images at L-band," in Proc. IGARSS, Boston, MA, Jul. 2008, pp. IV-145-IV-148.

[42] S. Sauer, L. Ferro-Famil, A. Reigber, and E. Pottier, "Polarimetric dualbaseline InSAR building height estimation at L-band," IEEE Geosci. Remote Sens. Lett., vol. 6, no. 3, pp. 408-412, Jul. 2009.

[43] E. R. Ferrara and T. M. Parks, "Direction finding with an array of antennas having diverse polarizations," IEEE Trans. Antennas Propag., vol. 31, no. 2, pp. 231-236, Mar. 1983.

[44] R. Horn, "The DLR airborne SAR project E-SAR," in Proc. IGARSS, Lincoln, May 1996, vol. 3, pp. 1624-1628.

[45] E. Rodriguez and J. M. Martin, "Theory and design of interferometric synthetic aperture radars," Proc. Inst. Elect. Eng. F Radar Signal Process., vol. 139, no. 2, pp. 147-159, Apr. 1992.

[46] P. Stoica and R. Moses, Introduction to Spectral Analysis. Englewood Cliffs, NJ: Prentice-Hall, 1997.

[47] G. Bienvenu, "Influence of the spatial coherence of the background noise on high resolution passive methods," in Proc. Int. Conf. Acoust., Speech, Signal Process., 1979, pp. 306-309.

[48] S. Sauer, L. Ferro-Famil, A. Reigber, and E. Pottier, "3D urban remote sensing using spectral analysis techniques applied to L-band dual-baseline POL-InSAR images," presented at the Eur. Conf. Synthetic Aperture Radar (EUSAR), Friedrichshafen, Germany, Jun. 2008.

[49] R. K. Raney, "SAR response to partially coherent phenomena," IEEE Trans. Antennas Propag., vol. 28, no. 6, pp. 777-787, Nov. 1980.

[50] G. Franceschetti, A. Iodice, D. Riccio, and G. Ruello, "SAR raw signal simulation for urban structures," IEEE Geosci. Remote Sens. Lett., vol. 41, no. 9, pp. 1986-1995, Sep. 2003.

[51] S. Sauer, "Télédétection SAR interférometrique de zones urbaines en bande $\mathrm{L}$ à partir de l'utilisation des techniques d'analyse spectrale polarimétriques et multi-bases, (Interferometric SAR remote sensing of urban areas at L-band using multibaseline and polarimetric spectral analysis techniques)," Ph.D. dissertation, Univ. Rennes 1, Rennes, France, Mar. 2008

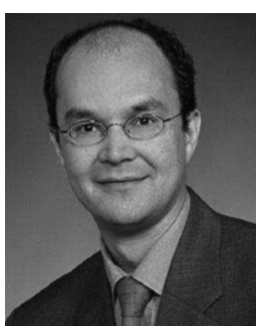

Stefan Sauer received the Diploma degree in mathematics from Berlin University of Technology, Berlin, Germany, in 2003 and the Ph.D. degree in signal processing and telecommunication from the University of Rennes 1, Rennes, France, in 2008.

From 2004 to 2008, he was a Research Engineer with the SAR Polarimetry, Holography, Interferometry and Radargrammetry Team, Institute of Electronics and Telecommunications of Rennes, University of Rennes 1, where he worked in the field of polarimetric spectral analysis techniques for processing polarimetric multibaseline interferometric synthetic aperture radar (SAR) data. Since 2008, he has been with the Microwaves and Radar Institute, German Aerospace Center (DLR), Oberpfaffenhofen, Germany, as a Research Scientist working in the field of SAR processing, SAR polarimetry, and multibaseline interferometry. His research interests include algorithms for processing polarimetric and multibaseline interferometric SAR measurements with applications in urban and forest remote sensing.

Dr. Sauer was the recipient of the second prize of the Student Paper Competition of the 2007 IEEE International Geoscience and Remote Sensing Symposium.

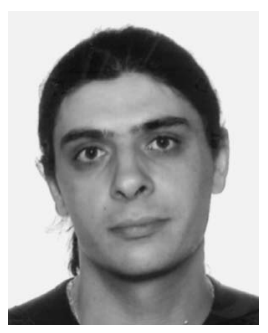

Laurent Ferro-Famil (S'99-A'00-M'04) received the Laurea degree in electronics systems and computer engineering, the M.S. degree in electronics, and the Ph.D. degree from the University of Nantes, Nantes, France, in 1996, 1996, and 2000, respectively.

In 2001, he became an Assistant Professor and, since 2007, he has been an Associate Professor with the University of Rennes 1, Rennes, France, where he is currently a Member of the Radar Polarimetry Remote Sensing Group, Institute of Electronics and Telecommunications of Rennes. His current activities in education are concerned with analog electronics, digital communications, microwave theory, and polarimetric synthetic aperture radar (SAR) remote sensing. He is particularly interested in polarimetric SAR signal processing, radar polarimetry theory, and natural media remote sensing using multibaseline PolInSAR data, with application to classification, electromagnetic scattering modeling and physical parameter retrieval, time-frequency analysis, and 3-D reconstruction of environments.

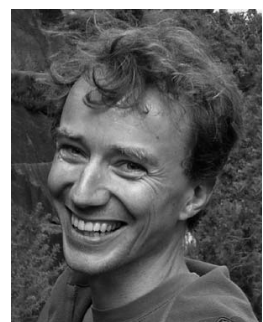

Andreas Reigber (M'02-SM'10) was born in Munich, Germany, in 1970. He received the Diploma degree in physics from the University of Constance, Konstanz, Germany, in 1997, the Ph.D. degree from the University of Stuttgart, Stuttgart, Germany, in 2001, and the Habilitation from Berlin University of Technology, Berlin, Germany, in 2008.

From 1996 to 2000, he was with the Microwaves and Radar Institute, German Aerospace Center (DLR), Oberpfaffenhofen, Germany, where he worked in the field of polarimetric synthetic aperture radar (SAR) tomography. In 2001, he joined the Antenna, Radar and Telecom Laboratories, University of Rennes 1, Rennes, France, as a Postdoctoral Researcher on radar polarimetry and polarimetric interferometry. From 2002 to 2007, he was a Research Associate with the Computer Vision and Remote Sensing Laboratories, Berlin University of Technology, Berlin. Since 2008, he has been with the Microwaves and Radar Institute, DLR, where he is currently the Head of the SAR Technology Department and directing the airborne SAR activities of the institute. His current main research interests are the various aspects of multimodal SAR, like SAR interferometry, SAR polarimetry, SAR tomography, and time-frequency analyses, and also the application of computer vision and machine learning approaches in remote sensing.

Dr. Reigber was the recipient of the 2000 European Conference on Synthetic Aperture Radar Student Prize Paper Award for an article on SAR remote sensing of forests, the IEEE TRANSACTIONS ON GEOSCIENCE AND REMOTE SENSING (TGRS) Transactions Prize Paper Award in 2001 for a work on polarimetric SAR tomography, and the IEEE TGRS LETTERS Prize Paper Award in 2006 for a work on multipass SAR processing.

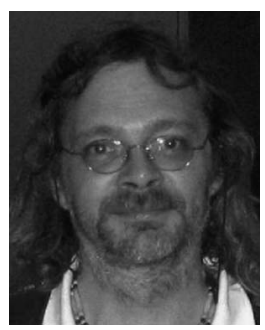

Eric Pottier (M'95-SM'06-F'11) received the M.Sc. and Ph.D. degrees in signal processing and telecommunication from the University of Rennes 1, Rennes, France, in 1987 and 1990, respectively, and the Habilitation from the University of Nantes, Nantes, France, in 1998.

Since 1999, he has been a full Professor with the University of Rennes 1, where he is currently the Deputy Director of the Institute of Electronics and Telecommunications of Rennes (IETR-CNRS UMR 6164) and also the Head of the SAR Polarimetry, Holography, Interferometry and Radargrammetry SAPHIR Team, Image and Remote Sensing Group. Since 1989, he has supervised more than 30 research students to graduation (Ph.D.) in radar polarimetry covering areas from theory to remote sensing applications. He has chaired and organized 35 sessions in international conferences and was a Member of the Technical and Scientific Committees of 32 international symposium or conferences. He has been invited to present 46 presentations in international conferences. $\mathrm{He}$ has published a book coauthored with Dr. J. S. Lee in 2009, nine publications in books, and more than 50 papers in refereed journals and has presented more than 330 papers during international conferences, symposiums, and workshops. $\mathrm{He}$ has presented advanced courses and seminars on radar polarimetry to a wide range of organizations (DLR, NASDA, JRC, RESTEC, and IECAS) and events (ISAP2000, EUSAR04-06-10, NATO-04-06, PolInSAR05-11, JAXA06, and IGARSS03-05-07-08-09-10). His current activities of research and education are centered on the topics of analog electronics, microwave theory, and radar imaging with emphasis in radar polarimetry. His research covers a wide spectrum of areas, from radar image processing [synthetic aperture radar (SAR) and inverse SAR], polarimetric scattering modeling, and supervised/unsupervised polarimetric segmentation and classification to fundamentals and basic theory of polarimetry.

Dr. Pottier was the recipient of the Best Paper Award at the Third European Conference on Synthetic Aperture Radar, the 2007 IEEE GRS-S PRIZE PAPER AWARD, AND THE 2007 IEEE GRS-S EDUCATION AWARD "IN RECOGNITION OF HIS SIGNIFICANT EDUCATIONAL CONTRIBUTIONS TO Geoscience And Remote Sensing." 